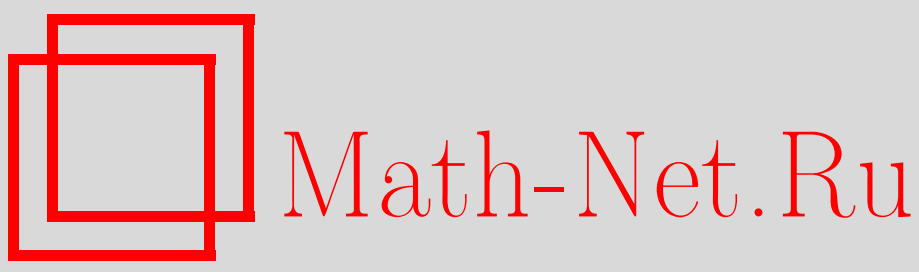

В. В. Филиппов, О существовании периодических решений уравнений с сильно растущей главной частью, Матем. сб., 2002, том 193, номер 11, 139-160

DOI: https://doi.org/10.4213/sm695

Использование Общероссийского математического портала Math-Net.Ru подразумевает, что вы прочитали и согласны с пользовательским соглашением http: //www. mathnet.ru/rus/agreement

Параметры загрузки:

IP : 44.207 .124 .84

26 апреля 2023 г., $12: 13: 33$ 
УДК 517.911

\section{В.В. Филиппов}

\section{О существовании периодических решений уравнений с сильно растущей главной частью}

Продолжается распространение теории краевых задач на обыкновенные дифоференциальные уравнения и включения с разрывньми правьми частями, основанное на построении нового варианта метода сдвига вдоль траекторий. В статье развивается аппарат доказательства существования периодических решений для уравнений второго порядка с суперлинейно растущими членами.

Библиография: 16 названий.

При доказательстве сушествования периодического решения для уравнения $x^{\prime \prime}+g(x)=h(t)$ ситуации, когда функция $g$ растет линейно, т.е. когда

$$
\alpha<\liminf _{|x| \rightarrow \infty} \frac{g(x)}{x} \leqslant \limsup _{|x| \rightarrow \infty} \frac{g(x)}{x}<\beta,
$$

и когда рост функции $g$ быстрее линейного, т.е. когда

$$
\lim _{|x| \rightarrow \infty} \frac{g(x)}{x}=\infty,
$$

сильно различаются.

Мы рассмотрим второй случай. Взяв за основу известные результаты на эту тему (см. [1]), мы освободим связанньй с этой задачей принцип продолжения по параметру от использования метода Лерэ-Шаудера, перейдя на развиваемый нами вариант метода сдвига вдоль траекторий [2]-[4].

Мы нацелены на анализ связей геометрических свойств решений, используемых в доказательствах подобных утверждений. Переход в рассуждениях от манипулирования правой частью к обсуждению геометрических свойств решений разделяет вопросы, относящиеся к теории задачи Коши, и вопросы, относящиеся к геометрическому содержанию исследуемой проблемы. Это соответствует общей идеологии подхода [5]-[9] к построению теории обькновенных дифференциальных уравнений. За счет ухода от обсуждения вопросов, относящихся к теории задачи Коши, мы очень сильно расширяем рассматриваемый класс уравнений в плане общих свойств правой части (непрерьвность, условия Каратеодори, Дэви и т. д.). Мы продолжаем распространение теории краевых задач на уравнения и включения с разрывной правой частью.

Напомним некоторые понятия и обозначения теории [5]-[8]. Рассматриваются уравнения и включения, правые части которых определены на открытом подмножестве $U$ произведения $\mathbb{R} \times \mathbb{R}^{n}$. Мы не предполагаем ничего относительно свойств

Работа вьполнена при поддержке Российского фонда фундаментальных исследований (грант № 01-01-00744).

(C) В.В. Филиппов 2002 
правых частей - ни непрерьвности, ни вьполнения условий Каратеодори. Символ $R(U)$ обозначает множество всех множеств функций, определенных на отрезках и одноточечных подмножествах и отрезках действительной прямой, которые замкнуты относительно операций 1) перехода к функции с меньшей областью определения и 2) составления взаимного продолжения из двух функций, совпадающих на непустом пересечении областей определения. Ясно, что при любом более-менее разумном определении решения (но в духе Каратеодори) множество решений любого уравнения или включения будет удовлетворять этим двум комбинаторным условиям, т.е. множество решений этого уравнения или включения обязательно будет членом множества $R(U)$. Следуя [5]-[8] в изложении общей теории обыкновенных дифференциальных уравнений, мы переходим затем на аксиоматический уровень, когда вместо обычного требования типа непрерывности правых частей рассматриваемых уравнений или выполнения условий Каратеодори мы накладываем на множества решений условия типа непрерывности зависимости решений от начальных условий (см. далее условие $(c)$ ), локального существования решения задачи Коши (см. далее условие $(e))$ и т. д. Сказанное легко понимается, когда выполнено условие единственности решения задачи Коши и когда все обсуждаемые решения определены на всем рассматриваемом временном отрезке. В общем случае требуются дополнительные обсуждения. В частности (при отсутствии единственности решения задачи Коши), непрерывность расширяется до полунепрерывности сверху. Равномерная сходимость функций получает более приспособленную для обсуждения более широких связей интерпретацию в виде сходимости относительно расстояния Хаусдорфа между графиками функций. Это позволяет рассматривать одновременно функции с различными (но компактными) областями определения. При этом названные вьше свойства (пространства решений $Z$ ) выглядят следующим образом:

$(e)$ для любой точки $(t, y)$ множества $U$ найдется такая функция $z$, принадлежашая $Z$, что $^{1} t \in\langle\pi(z)\rangle$ и $z(t)=y$ (т.е. задача Коши для пространства $Z$ с начальньп условием $z(t)=y$ ) имеет решение,

$(c)$ для любого компактного подмножества $K$ множества $U$ множество $Z_{K}$ компактно.

Свойство $(c)$ является псевдонимом непрерывности зависимости решений задачи Коши от начальных условий (символ $Z_{K}$ обозначает множество всех тех элементов пространства $Z$, графики которых лежат в $K$; имеется в виду компактность относительно расстояния Хаусдорфа между графиками).

В рассматриваемом круге вопросов для нас будет существенньм также требование гомологической тривиальности множеств решений, начинающихся в одной точке, а именно свойство

$(h)$ для любой точки $\left(a, y_{0}\right) \in U$, множество $S_{Z}\left(y_{0} ; a, b\right)$ ациклично, т.е.

$$
H_{i}\left(S_{Z}\left(y_{0} ; a, b\right) ; \mathbb{Q}\right)= \begin{cases}\mathbb{Q} & \text { при } i=0 ; \\ 0 & \text { при } i \neq 0\end{cases}
$$

для любой точки $\left(a, y_{0}\right) \in U$ и любого отрезка $[a, b] \subseteq \mathbb{R}$, удовлетворяющего условию:

$$
[a, b] \subseteq \pi(z) \text { для любого решения задачи Коши } z \in Z^{+}, z(a)=y_{0} \text {. }
$$

\footnotetext{
${ }^{1}$ Символ $\langle M\rangle$ обозначает внутренность множества $M$.
} 
Пусть $*-$ некоторый список свойств указанного типа. Символ $R_{*}(U)$ обозначает множество всех членов множества $R(U)$, удовлетворяющих всем условиям из списка $*$.

На множестве $R_{c}(U)$ естественньм образом вводится топология, адекватная непрерывности зависимости решений от параметров уравнения. Именно она используется далее, например, в условии (1.2). Рассматривая семейство уравнений $y^{\prime}=f(t, y, \alpha)$, мы интерпретируем непрерывность зависимости решений от параметра $\alpha$ следующим образом. Мы требуем, чтобы для любой последовательности индексов $\left\{\alpha_{i}, i=1,2, \ldots\right\}$, сходящейся к некоторому значению $\alpha_{0}$, пространства $Z_{i}$ решений соответствующих уравнений $y^{\prime}=f\left(t, y, \alpha_{i}\right)$ сходились к пространству $Z_{0}$ решений уравнения $y^{\prime}=f\left(t, y, \alpha_{0}\right)$ в смысле выполнения следующего условия:

для любого компактного подмножества $K$ множества $U$ и любой последовательности функций $\left\{z_{j} \in Z_{j}, j \in A\right\}$, графики которых лежат в компакте $K$, найдутся функция $z \in Z$ и подпоследовательность последовательности $\left\{z_{j}, j \in A\right\}$, сходящаяся к функции $z$.

Это свойство остается содержательным и при рассмотрении стационарной последовательности $Z_{j} \equiv Z$. В этом случае оно приобретает вид приведенного выше условия $(c)$.

Если вьполнено условие единственности решения задачи Коши и все обсуждаемые решения определены на всем рассматриваемом временном отрезке, то приведенные условия приобретают общепонятный, обшеизвестный вид. Более детальное исследование подобных свойств можно найти в [3]-[8] (см. также [10] и [11]).

Символ $P^{T}(U)$ обозначает класс $T$-периодических пространств из $R(U)$, т.е. если $Z \in P^{T}(U)$ и $z \in Z$, то функции $z(t \pm T)$ также принадлежат $Z$ (см. [6; $\S$ IX.4] или $[8 ; \S 15.4])$.

\section{§1. Продолжение по параметру}

Следующая теорема содержит принцип продолжения по параметру, адекватньй основной обсуждаемой в этой статье ситуации и дополняющий не метод Лерэ-Шаудера, а метод сдвига вдоль траекторий. Она является вариантом теоремы 4.2 из [2] (в [2] $T=1$; доказательство повторяется дословно с оговоркой относительно этой замены, либо нашу теорему 1.1 можно считать формальньм следствием теоремы 4.2 в [2] ввиду гомеоморфности отрезков $[0,1]$ и $[0, T])$.

TEOPEMA 1.1. Пусть

(1.1) $U=\mathbb{R} \times \mathbb{R}^{n} u 0<T<\infty$;

(1.2) $-\infty<c<d<\infty$ и при любом $\lambda \in[c, d]$ пространство $Z_{\lambda}$ принадлежит классу $P_{c е h}^{T}(U)$, отображение $\varphi:[c, d] \rightarrow P_{c е h}^{T}(U)$, заданное формулой $\varphi(\lambda)=Z_{\lambda}$, непрерывно;

(1.3) $r>0, S_{r}=\left\{u: u \in \mathbb{R}^{n},\|u\|=r\right\}, K_{r}=\left\{u: u \in \mathbb{R}^{n},\|u\| \leqslant r\right\}$;

(1.4) ecлu $z \in Z_{c}^{+}, \inf \pi(z)=0 u z(0) \in K$, mo $\pi(z) \supseteq[0, T]$;

(1.5) $z(T) \neq z(0)$ для любой функиии $z$ из множсества $X=\left\{z: z \in Z_{c}\right.$, $\left.\pi(z)=[0, T], z(0) \in S_{r}\right\}$ и степень сдвига $z \mapsto z(T)-z(0)$ множества $X$ отлична от нуля;

(1.6) множество $D=\left\{(\lambda, z): \lambda \in[c, d], z \in Z_{\lambda}, \pi(z)=[0, T], z(0)=z(T)\right\}$ $\left(\subseteq[c, d] \times C\left([0, T], \mathbb{R}^{n}\right)\right)$ распадается в обвединение дискретного семейства компактов. 
Тогда найдется такая функиия $z \in Z_{d}$, ито $\pi(z)=[0, T] u z(T)=z(0)$.

В формулировке теоремы 1.1 принадлежность пространства $Z_{\lambda}$ классу $R_{c e h}(U)$ означает, грубо говоря, успешность распространения на соответствуюшее уравнение $x^{\prime}=f(t, x, \lambda)$ теории задачи Коши вплоть до оценки гомологических свойств множеств решений (см. подробности в [2], [5]-[8]). Непрерывность отображения $\varphi$ означает непрерывность зависимости решений уравнения $x^{\prime}=f(t, x, \lambda)$ от параметра $\lambda$.

Следующая теорема содержит другой вариант принципа продолжения по параметру также для сдвига вдоль траекторий. Она является вариантом теоремы 2.1 из [3] (в [3] понятие степени не использовалось и говорилось о соответствующем гомоморфизме; тривиальные леммы 2.6 и 2.7 в [4] устраняют это расхождение, кроме того теорема 1.2 является формальньм следствием теорем 6.1 и 6.2 в [12]). Она более, чем теорема 1.1, в смысле строения сдвигаемого множества (условия $(1.5)$ и (1.10)) следует идеологии принципа продолжения по параметру в методе Лерэ-Шаудера.

ТЕОРЕМА 1.2. Пусть в обозначениях (1.1), (1.2)

(1.7) множсество $W$ является открытым ограниченным подмножеством пространства $C\left([0, T], \mathbb{R}^{n}\right)$;

(1.8) для $\lambda \in[c, d] H_{\lambda}=\{z: z \in \varphi(\lambda) \cap[W]\} u G_{\lambda}=\{z: z \in \varphi(\lambda) \cap W\}$;

(1.9) при любом $\lambda \in[c, d] z(0) \neq z(T)$ для любой функиии zиз $H_{\lambda} \backslash G_{\lambda}$;

(1.10) степень сдвига $z \mapsto z(T)-z(0)$ множества $Z_{c} \cap\left(H_{c} \backslash G_{c}\right)$ отлична от нуля.

Тогда степень сдвига $z \mapsto z(T)-z(0)$ множества $Z_{d} \cap\left(H_{d} \backslash G_{d}\right)$ также отлична от нуля и найдется такая функция $z \in Z_{d}$, что $\pi(z)=[0, T] u$ $z(T)=z(0)$.

\section{§2. Поведение траекторий}

Пусть в обозначениях (1.1)

(2.1) $Z \in P_{c e h}^{T}(U)$ - пространство решений дифференциального включения второго порядка $x^{\prime \prime} \in F\left(t, x, x^{\prime}\right)$. Т.е. речь идет о пространстве решений соответствующей системы

$$
\left\{\begin{array}{l}
x^{\prime}=y, \\
y^{\prime} \in F(t, x, y)
\end{array}\right.
$$

первого порядка. При этом мы не накладываем ограничений на тип самой функции $F$ (см. [9]). Принадлежность $Z \in P^{T}(U)$ практически равносильна $T$-периодичности функции $F$ по $t$.

Для нас будет существенно наличие следующего свойства решений этого включения.

(2.2) Если $z=(x, y) \in Z^{+}$и $\lim _{t \rightarrow \sup \pi(z)}|y(t)|=\infty$, то $\lim _{t \rightarrow \sup \pi(z)}|x(t)|=$ $\infty$. Если $z=(x, y) \in Z^{-}$и $\lim _{t \rightarrow \inf \pi(z)}|y(t)|=\infty$, то $\lim _{t \rightarrow \inf \pi(z)}|x(t)|=\infty$.

То есть, грубо говоря, если абсциссы точек некоторой траектории ограничены, то она не уходит на бесконечность (рис. 1).

ЛЕмма 2.1. Пусть в обозначениях (1.1) $и$ (2.1) для некоторого $R>1$ и для любъх отрезков $I, J \subseteq \mathbb{R}$ найдутся такие интегрируемые функиии $\varphi_{1}: I \rightarrow[0, \infty)$ и $\varphi_{2}: J \rightarrow[0, \infty)$, что множество $F(t, x, y)$ лежит в отрезке 


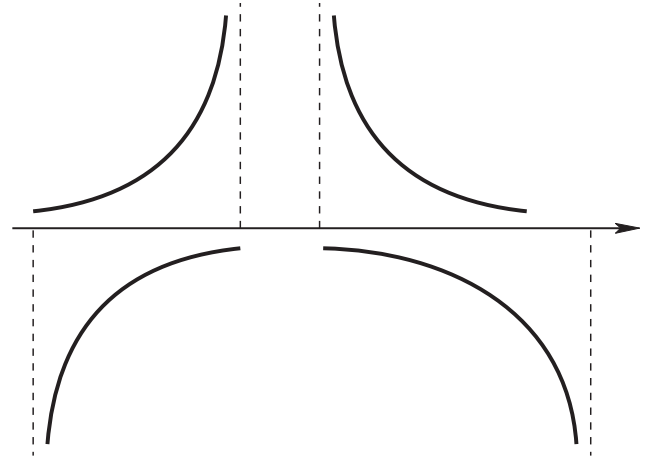

Рис. 1. "Запрещенные" траектории

$\left[-\varphi_{1}(x)-\varphi_{2}(t), \varphi_{1}(x)+\varphi_{2}(t)\right]$ nри всех $t \in J, x \in I$ u $|y|>R$. Тогда условие (2.2) выполнено.

ДокАЗАТЕЛЬСтво. Условие (2.2) распадается на случаи в соответствии со знаками функций $x$ и $y$ и принадлежностью $z \in Z^{+}$или $z \in Z^{-}$. Пусть $z=(x, y) \in Z^{+}$ и $\lim _{t \rightarrow \sup \pi(z)} y(t)=\infty$. Зафиксируем такое число $t_{0}>0$, что $y(t)>R$ при $t \geqslant t_{0}$. Так как $x^{\prime}(t)=y(t)>R>1$ при $t \geqslant t_{0}$, то функция $x(t)$ является здесь возрастающей. Ввиду возрастания функции $x$ конечный или бесконечный предел $\lim _{t \rightarrow \sup \pi(z)} x(t)$ сушествует. Предположим, что $\lim _{t \rightarrow \sup \pi(z)} x(t) \neq \infty$, т.е. что предел $c_{0}=\lim _{t \rightarrow \sup \pi(z)} x(t)$ строго меньше $\infty$. Тогда $\sup \pi(z) \leqslant c_{1}$, где $c_{1}=t_{0}+\left(c_{0}-x\left(t_{0}\right)\right)<\infty$. Для отрезков $I=\left[x\left(t_{0}\right), c_{0}\right] \subseteq \mathbb{R}$ и $J=\left[t_{0}, c_{1}\right] \subseteq \mathbb{R}$ выбираем такие интегрируемые функции $\varphi_{1}: I \rightarrow[0, \infty)$ и $\varphi_{2}: J \rightarrow[0, \infty)$, что $F(t, x, y)$ лежит в отрезке $\left[-\varphi_{1}(x)-\varphi_{2}(t), \varphi_{1}(x)+\varphi_{2}(t)\right]$. Тогда

$$
\begin{aligned}
\left|y(t)-y\left(t_{0}\right)\right| & =\left|\int_{t_{0}}^{t} y^{\prime}(s) d s\right| \leqslant \int_{t_{0}}^{t}\left(\varphi_{1}(x(s))+\varphi_{2}(s)\right) d s \\
& \leqslant \int_{t_{0}}^{t} \varphi_{1}(x(s)) x^{\prime}(s) d s+\int_{t_{0}}^{t} \varphi_{2}(s) d s \leqslant \int_{I} \varphi_{1}(u) d u+\int_{J} \varphi_{2}(s) d s .
\end{aligned}
$$

Но это справедливо для любого $t \in \pi(z) \cap\left[t_{0}, \infty\right)$, поэтому

$$
\lim _{t \rightarrow \sup \pi(z)} y(t) \leqslant y\left(t_{0}\right)+\int_{I} \varphi_{1}(u) d u+\int_{J} \varphi_{2}(s) d s<\infty .
$$

(Последняя оценка справедлива в силу интегрируемости функций $\varphi_{1}$ и $\varphi_{2}$.) Но это противоречит исходному предположению, что $\lim _{t \rightarrow \sup } \pi(z) y(t)=\infty$.

Таким образом, сделанное нами предположение, что $\lim _{t \rightarrow \sup \pi(z)} x(t) \neq \infty$, привело нас к противоречию, что и дает требуемое. Остальные случаи рассматриваются аналогично. Лемма доказана.

ЛЕмма 2.2. Пусть выполнены условия (1.1), (1.2) и каждое из пространств $Z_{\lambda}, \quad \lambda \in[c, d]$, удовлетворяет условиям (2.1) и (2.2). Пусть $-\infty<p_{0}<p_{1}<\infty$ u $R>0$. Тогда найдется такое число $m>0$, что если $z=(x, y) \in Z_{\lambda}^{+}, \lambda \in[c, d], 0<y(\inf \pi(z)) \leqslant R, t_{0} \in \pi(z), y(t)>0 u$ $p_{0} \leqslant x(t) \leqslant p_{1}$ nрu $t \in\left[\inf \pi(z), t_{0}\right)$, то $y\left(t_{0}\right)<m$ (рис. 2 ). 
ДокаЗАТЕльство. Допустим противное. Тогда для любого $m=1,2, \ldots$ найдутся такая функция $z_{m}=\left(x_{m}, y_{m}\right) \in Z_{\lambda_{m}}^{+}$и такое число $t_{m} \in \pi(z)$, что $0<y_{m}\left(\inf \pi\left(z_{m}\right)\right) \leqslant R, y\left(t_{m}\right) \geqslant R+m$ и $y_{m}(t)>0, p_{0} \leqslant x_{m}(t) \leqslant p_{1}$ при $t \in\left[\inf \pi\left(z_{m}\right), t_{m}\right)$.

В силу $T$-периодичности рассматриваемых пространств, не ограничивая общности, мы можем предполагать, что $0 \leqslant \inf \pi\left(z_{m}\right) \leqslant T$. Кроме того, нам достаточно, очевидно, рассмотреть случай, когда $y_{m}(t) \geqslant 1$ при $t \in\left[\inf \pi\left(z_{m}\right), t_{m}\right)$. При этом мы имеем $t_{m} \leqslant T+p_{1}-p_{0}$.

В силу компактности отрезка $[c, d]$ мы можем предположить дополнительно, что последовательность $\left\{\lambda_{m}, m=1,2, \ldots\right\}$ сходится к некоторой точке $\lambda_{0} \in[c, d]$. По лемме 10.4.1r из [8] существуют такая функция $z=(x, y) \in Z_{\lambda_{0}}^{+}$и такая подпоследовательность $\left\{z_{m}, m \in A\right\}$ последовательности $\left\{z_{m}, m=1,2, \ldots\right\}$, что для любой точки $t \in \pi(z)$ справедливо следующее: $\pi\left(z_{m}\right) \ni t$, начиная с некоторого $m=m_{0}$, и последовательность $\left\{\left.z_{m}\right|_{(-\infty, t] \cap \pi\left(z_{m}\right)}, m \in A\right\}$ сходится к функции $\left.z\right|_{(-\infty, t] \cap \pi(z)}$. В частности, $\inf \pi(z)=\lim _{m \rightarrow \infty, m \in A} \inf \pi\left(z_{m}\right) \in[0, T]$.

В силу того, что $\lim _{m \rightarrow \infty} y_{m}\left(t_{m}\right)=\infty$, и в силу сказанного в предыдущем абзаце для функции $z$ мы имеем $\pi(z) \subseteq\left[0, T+p_{1}-p_{0}\right] \lim _{t \rightarrow \sup } \pi(z) y(t)=\infty$. Но это противоречит условию (2.2). Таким образом, сделанное допущение привело к противоречию, что и дает требуемое. Лемма доказана.

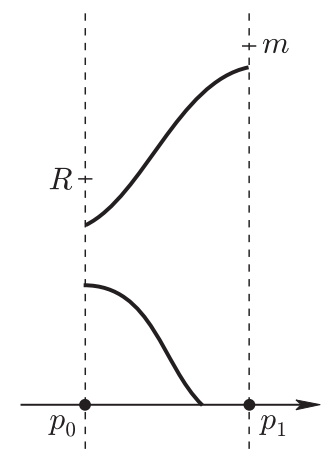

Рис. 2

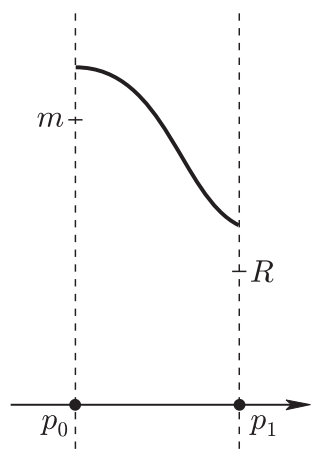

Рис. 3

ЛЕмма 2.3. Пусть выполнень условия (1.1), (1.2) и кажсдое из пространств $Z_{\lambda}, \quad \lambda \in[c, d]$, удовлетворяет условиям (2.1) и (2.2). Пусть $-\infty<p_{0}<p_{1}<\infty$ u $R>0$. Тогда найдется такое число $m>0$, что если $z=(x, y) \in Z_{\lambda}^{+}, p_{0} \leqslant x(\inf \pi(z)) \leqslant p_{1} u y(\inf \pi(z))>m$, то найдется такое $t_{0} \in \pi(z)$, что $x\left(t_{0}\right)=p_{1} u y(t)>R, p_{0} \leqslant x(t) \leqslant p_{1}$ nрu $t \in\left[\inf \pi(z), t_{0}\right]$ (рис. 3 ).

ДокАЗАтЕльство. Утверждение достаточно доказать для больших $R$, поэтому, не ограничивая общности, мы можем предположить, что $R>1$.

Допустим, что доказываемое неверно. Тогда для любого $m=1,2, \ldots$ найдется такая функция $z_{m}=\left(x_{m}, y_{m}\right) \in Z_{\lambda_{m}}$, что $y\left(\sup \pi\left(z_{m}\right)\right)=R, y_{m}(t)>R$ и $p_{0} \leqslant x(t) \leqslant p_{1}$ при $t \in \pi\left(z_{m}\right)$ и, наконец, $y_{m}\left(\inf \pi\left(z_{m}\right)\right)>R+m$. В силу $T$-периодичности рассматриваемых пространств, не ограничивая общности, мы можем предполагать, что $0 \leqslant \inf \pi\left(z_{m}\right) \leqslant T$. В силу того, что $x^{\prime}(t)=y(t)>R>1$, мы имеем $\sup \pi\left(z_{m}\right) \leqslant T+p_{1}-p_{0}$.

Каждую из функций $z_{m}$ продолжим произвольно до функции $z_{m}^{*} \in Z_{\lambda_{m}}^{-}$. 
В силу компактности отрезка $[c, d]$ мы можем предположить дополнительно, что последовательность $\left\{\lambda_{m}, m=1,2, \ldots\right\}$ сходится к некоторой точке $\lambda_{0} \in[c, d]$. По лемме 10.4.11 из [8] сушествуют такая функция $z=(x, y) \in Z_{\lambda_{0}}^{-}$и такая подпоследовательность $\left\{z_{m}^{*}, m \in A\right\}$ последовательности $\left\{z_{m}^{*}, m=1,2, \ldots\right\}$, что для любой точки $t \in \pi(z)$ справедливо следующее: $\pi\left(z_{m}^{*}\right) \ni t$, начиная с некоторого $m=m_{0}$, и последовательность $\left\{\left.z_{m}^{*}\right|_{[t, \infty) \cap \pi\left(z_{m}^{*}\right)}, m \in A\right\}$ сходится к функции $\left.z\right|_{[t, \infty) \cap \pi(z)} \cdot$

В силу того, что $\lim _{m \rightarrow \infty} y_{m}\left(\inf \pi\left(z_{m}\right)\right)=\infty$, и в силу сказанного в предыдущем абзаце для функции $z$ мы имеем $\pi(z) \subseteq\left[0, T+p_{1}-p_{0}\right]$ и $\lim _{t \rightarrow \inf } \pi(z) y(t)=\infty$. Но это противоречит условию (2.2). Таким образом, сделанное допущение привело к противоречию, что и дает требуемое. Лемма доказана.

СлЕДСТВИЕ. Пусть выполнены условия (1.1), (1.2) и каждое из пространств $Z_{\lambda}, \lambda \in[c, d]$, удовлетворяет условиям (2.1) и (2.2). Тогда если $z_{k}=\left(x_{k}, y_{k}\right) \in Z_{\lambda_{k}}, \pi\left(z_{k}\right)=\left[s_{k}, t_{k}\right], x_{k}\left(s_{k}\right)=x_{k}\left(t_{k}\right)=0, x_{k}(t)>0$ nрu $t \in\left(s_{k}, t_{k}\right) u y_{k}\left(s_{k}\right) \rightarrow \infty n p u k \rightarrow \infty$, mo $\sup x_{k}\left(\left[s_{k}, t_{k}\right]\right) \rightarrow \infty$.

ЗАмЕчАНИЕ 2.1. В предположениях леммы 2.2 траектория $z(t)$ обязательно доходит либо до оси абсцисс, либо до вертикали $x=p_{1}$. В предположениях леммы 2.3 первый случай исключается и траектория $z(t)$ обязательно доходит до вертикали $x=p_{1}$.

ЛЕмма 2.4. Пусть выполнень условия (1.1), (1.2) и кажсдое из пространств $Z_{\lambda}, \lambda \in[c, d]$, удовлетворяет условиям (2.1) и (2.2). Пусть $-\infty<p_{0}<\infty, \varepsilon>0$ и $R>0$. Тогда найдется такое число $m>0$, что если $z=(x, y) \in Z_{\lambda}^{+}, x(\inf \pi(z))=p_{0}, y(\inf \pi(z))>m u y^{\prime}(t) \leqslant-\varepsilon$ почти при всех $t \in \pi(z)$, для которых $x(t) \geqslant p_{0}$, то найдется такое $s \in \pi(z)$, что $x(t) \geqslant p_{0}$ при всех $t \in[\inf \pi(z), s], x(s)=p_{0} u y(s)<-R$ (рис. 4).

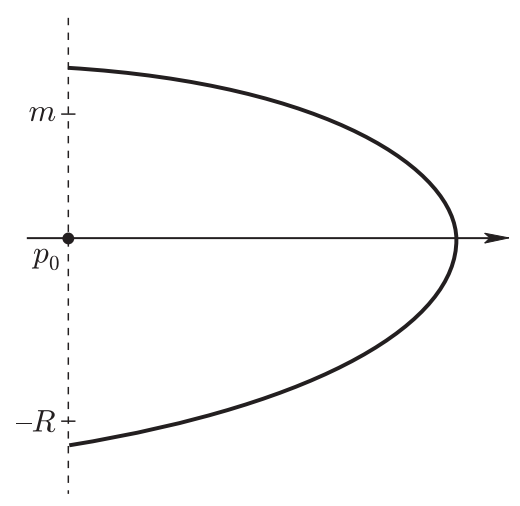

Рис. 4

ДоказАтельство. 1) Отметим прежде всего, что если $z=(x, y) \in Z_{\lambda}^{+}$, $x(\inf \pi(z))=p_{0}, y(\inf \pi(z))>0$ и $y^{\prime}(t) \leqslant-\varepsilon$ почти при всех $t \in \pi(z)$, для которых $x(t) \geqslant p_{0}$, то траектория $z(t)$ не позднее, чем через время $y(\inf \pi(z)) / \varepsilon$, перейдет через ось абсцисс в точке с абсциссой больше $p_{0}$. При некотором значении $t=t_{0}$ точка будет ниже оси абсцисс, и далее функции $x(t)$ и $y(t)$ будут убывать, поэтому не позднее, чем через время $x\left(t_{0}\right) /\left|y\left(t_{0}\right)\right|$, траектория $z(t)$ достигнет вертикали $x=p_{0}$. 
2) В силу 1) если мы допустим, что доказываемое неверно, то для любого $m=$ $1,2, \ldots$ найдется такая функция $z_{m}=\left(x_{m}, y_{m}\right) \in Z_{\lambda_{m}}$, что $x_{m}(t) \geqslant p_{0}$ при всех $t \in \pi(z), x_{m}\left(\inf \pi\left(z_{m}\right)\right)=x_{m}\left(\sup \pi\left(z_{m}\right)\right)=p_{0}, y_{m}(\inf \pi(z))>m, y_{m}^{\prime}(t) \leqslant-\varepsilon$ при всех $t \in \pi(z)$ и $y_{m}\left(\sup \pi\left(z_{m}\right)\right) \geqslant-R$.

В силу сказанного в 1) число $t_{m}=\sup \left\{t: t \in \pi\left(z_{m}\right), y_{m}(t)>0\right\}$ строго меньше $\sup \pi\left(z_{m}\right)$. Так как функция $y_{m}$ является убывающей $\left(y_{m}^{\prime}(t) \leqslant-\varepsilon\right)$, то $y_{m}(t)>0$ при $t<t_{m}, y_{m}\left(t_{m}\right)=0, y_{m}(t) \leqslant 0$ при $t>t_{m}$ и точка $t_{m}$ является точкой максимума для функции $x_{m}$. В частности, $y_{m}\left(\sup \pi\left(z_{m}\right)\right) \leqslant 0$ или, с учетом первоначального предположения относительно $y_{m}\left(\sup \pi\left(z_{m}\right)\right)$, мы имеем $y_{m}\left(\sup \pi\left(z_{m}\right)\right) \in[-R, 0]$. Поэтому $\sup \pi\left(z_{m}\right)-t_{m} \leqslant R / \varepsilon$ и $x_{m}\left(t_{m}\right) \leqslant p_{0}+R^{2} / \varepsilon$.

Теперь существование таких функций $z_{m}$ противоречит лемме 2.3 (с $p_{1}=p_{0}+$ $R^{2} / \varepsilon$ ) или следствию леммы 2.3. Лемма доказана.

ЗАМЕЧАниЕ 2.2. В силу рассуждений п. 1) доказательства в предположениях леммы 2.4 траектория $z(t)$ обязательно возвращается на вертикаль $x=p_{0}$, теперь ниже оси абсцисс.

ЛЕМма 2.5. Пусть выполнень условия (1.1), (1.2) и каждое из пространств $Z_{\lambda}, \quad \lambda \in[c, d]$, удовлетворяет условиям (2.1) и (2.2). Пусть $0<p_{0}<\infty, R>0, \varepsilon>0 u$

(2.3) ecлu $z_{k}=\left(x_{k}, y_{k}\right) \in Z_{\lambda_{k}}, \pi\left(z_{k}\right)=\left[s_{k}, t_{k}\right], x_{k}\left(s_{k}\right)=x_{k}\left(t_{k}\right)=p_{0}$, $x_{k}(t)>p_{0}$ nри $t \in\left[s_{k}, t_{k}\right]$, то $y_{k}^{\prime}(t) \leqslant-\varepsilon$ nочти при всех $t \in\left[s_{k}, t_{k}\right]$.

Тогда найдется такое число $m>0$, что если $z=(x, y) \in Z_{\lambda}^{+}, x(\inf \pi(z))=0$, $y(\inf \pi(z))>m$, то найдется такое $t_{0} \in \pi(z)$, что $x(t)>0$ при всех $t \in$ (inf $\left.\pi(z), t_{0}\right), x\left(t_{0}\right)=0 u y\left(t_{0}\right)<-R$.

ДоказАтЕльство (см. рис. 5). 1) Существует такое число $m_{1}>0$, что если $z=(x, y) \in Z_{\lambda}, 0 \leqslant x(t) \leqslant p_{0}$ при всех $t \in \pi(z), x(\inf \pi(z))=p_{0}, y(\inf \pi(z))<$ $-m_{1}$, то $y(t)<-R$ при всех $t \in \pi(z)$. Это следует из леммы 2.3 после замены переменных $(t, x, y) \mapsto(t,-x .-y)$. В силу замечания 2.1 все такие функции $z$ продолжимы до пересечения траектории с осью ординат.

2) Сушествует такое число $m_{2}>0$, что если функция $z=(x, y)$ принадлежит $Z_{\lambda}, x(t) \geqslant p_{0}$ при всех $t \in \pi(z), x(\inf \pi(z))=x(\sup \pi(z))=p_{0}, y(\inf \pi(z))>m_{2}$, то $y(\sup \pi(z))<-m_{1}$. Это следует из леммы 2.4 .

3) Сушествует такое число $m>0$, что если $z=(x, y) \in Z_{\lambda}, 0 \leqslant x(t) \leqslant p_{0}$ при всех $t \in \pi(z), x(\inf \pi(z))=0, y(\inf \pi(z))>m$, то $y(t)>m_{2}$ при всех $t \in \pi(z)$. Это следует из леммы 2.3. Как и в 1), все такие функции $z$ продолжимы до пересечения траектории с вертикалью $x=p_{0}$.

Проверка того, что при таком выборе выполняется требуемое условие, не составляет труда. Лемма доказана.

ЛЕмма 2.6. Пусть выполнены условия (1.1), (1.2) и каждое из пространств $Z_{\lambda}, \lambda \in[c, d]$, удовлетворяет условиям (2.1) и (2.2). Пусть $0<p_{0}<\infty$, $R>0, \varepsilon>0$ и выполнень условие (2.3) и условие

$\left(2.3^{\prime}\right)$ если $z_{k}=\left(x_{k}, y_{k}\right) \in Z_{\lambda_{k}}, \pi\left(z_{k}\right)=\left[s_{k}, t_{k}\right], x_{k}\left(s_{k}\right)=x_{k}\left(t_{k}\right)=-p_{0}$, $x_{k}(t)<-p_{0}$, mо $y_{k}^{\prime}(t) \geqslant \varepsilon$ почти при всех $t \in\left[s_{k}, t_{k}\right]$.

Тогда найдется такое число $m>0$, что если $z=(x, y) \in Z_{\lambda}^{+}, x(\inf \pi(z))=0$, $y(\inf \pi(z))>m$, то при некотором $t_{0} \in \pi(z)$ кривая $z(t), t \in\left[\inf \pi(z), t_{0}\right]$, совершает один оборот вокруг начала координат, $x\left(t_{0}\right)=0, y\left(t_{0}\right)>R$ (рис. 6 ). 


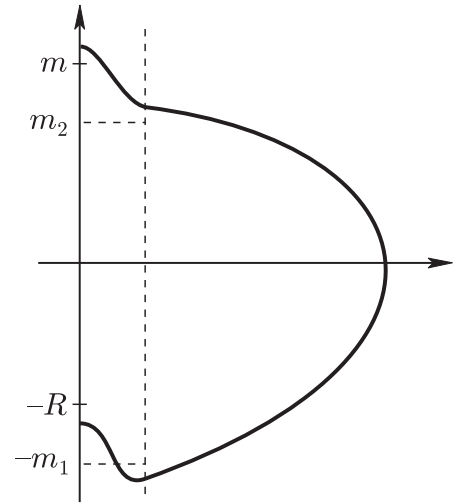

Рис. 5

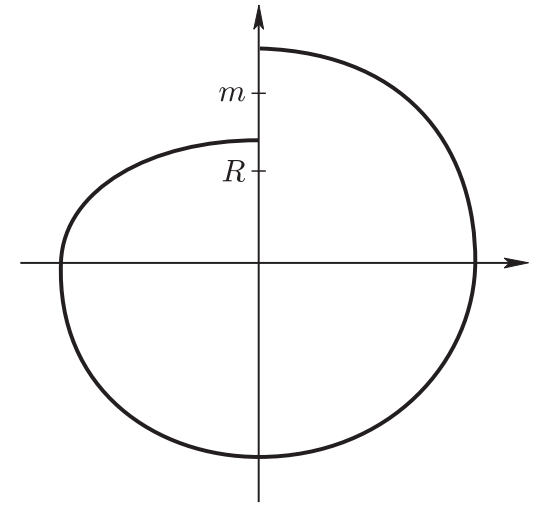

Рис. 6

ДоКАЗАТЕЛЬСТво аналогично доказательству леммы 2.5 со ссылкой на лемму 2.5 вместо использованных там ссылок. Лемма доказана.

ЛЕмма 2.7. Пусть выполнены условия (1.1), (1.2) и каждое из пространств $Z_{\lambda}, \lambda \in[c, d]$, удовлетворяет условиям (2.1) и (2.2). Пусть $0<p_{0}<\infty$, $\varepsilon>0$, выполнены условия (2.3) и $\left(2.3^{\prime}\right)$. Тогда

(2.4) для любъх $L>0$ и $l=1,2, \ldots$ найдется такое число $m>0$, что если $z=(x, y) \in Z_{\lambda}^{+}, x(\inf \pi(z))=0, y(\inf \pi(z))>m$, то при некотором $t_{0} \in \pi(z)$ кривая $z(t), t \in\left[\inf \pi(z), t_{0}\right]$, возвращается на положсительный луч оси ординат и совершает $l$ оборотов вокруг начала координат, пересекая полохсительный луч оси ординат в точках с ординатой больше L.

ДОКАЗАТЕЛЬСТВО сводится к очевидной индукции со ссылкой на лемму 2.6. Лемма доказана.

ЛЕМма 2.8. Пусть выполнень условия (1.1), (1.2) и каждое из пространств $Z_{\lambda}, \lambda \in[c, d]$, удовлетворяет условиям (2.1) и (2.2). Пусть L луч, начинающийся в начале координат. Пусть $R>0, p_{0}>0, \varepsilon>0$, $l=1,2, \ldots$, выполнены условия (2.3) и (2.3'). Тогда множсество $M$ функций $z=(x, y) \in Z_{\lambda}, \lambda \in[c, d], \sup \pi(z)-\inf \pi(z) \leqslant T$, для которых соответствующие траектории начинаются на луче $L$ и точки пересечения траекторий $c$ лучом $L$ удалены от начала координат на расстояние, не превосходящее $R$, является ограниченным.

ДоКАЗАТЕЛьСТво распадается на случаи в соответствии с расположением луча $L$. Мы ограничимся рассмотрением одного из возможных случаев, а именно мы будем предполагать, что $L$ - положительный луч оси ординат.

1) По лемме 2.2 существует такое число $R_{1}$, что если $z=(x, y) \in Z_{\lambda}, \lambda \in[c, d]$, $0<y(\inf \pi(z)) \leqslant R, y(t)>0$ и $-p_{0} \leqslant x(t) \leqslant p_{0}$ при всех $t \in \pi(z)$, то $y(t)<R_{1}$ при Bcex $t \in \pi(z)$.

В области $x>p_{0}$ ординаты точек кривых $z(t), z \in M$, тем более менњше $R_{1}$.

2) Абсциссы точек кривых $z(t)$ не превосходят $R_{2}=p_{0}+R_{1}^{2} / \varepsilon$.

3 ) Найдется такая константа $R_{3}>0$, что ординаты точек кривых $z(t)$ в правой полуплоскости $x \geqslant-p_{0}$ не менњше $-R_{3}$. Это следует из леммы 2.2 (см. также п. 1) доказательства леммы 2.5). В области $x<-p_{0}$ ординаты точек кривых $z(t)$, $z \in M$, тем более больше $-R_{3}$. 
4) Абсциссы точек кривых $z(t)$ не меньше $R_{4}=-p_{0}-R_{3}^{2} / \varepsilon$.

$5)$ Найдется такая константа $R_{5}>0$, что ординаты точек кривых $z(t)$ в правой полуплоскости $x \leqslant 0$ не больше $R_{5}$. Это следует из леммы 2.2 (см. также 3$\left.)\right)$.

Другие случаи рассматриваются аналогично. Лемма доказана.

\section{§ 3. Рост правой части и скорость вращения}

Пусть

$(3.1)$ функция $\gamma:[0, \infty) \rightarrow \mathbb{R}$ локально интегрируема (т.е. интегрируема на любом отрезке, лежашем в $[0, \infty))$,

(3.2) $\liminf _{x \rightarrow \infty} \frac{\gamma(x)}{x}>0$,

(3.3) для любой последовательности $\mu_{i} \rightarrow 0, \mu_{i}>0$, последовательность функций $g_{i}(x)=\mu_{i} \gamma\left(x / \mu_{i}\right)$ сходится на отрезке $[0,1]$ к бесконечности в смысле выполнения следуюшего условия: для любого $R>0$ мера множества $\{x: x \in[0,1]$, $\left.g_{i}(x)<R\right\}$ стремится к нулю.

ЗАмЕчАниЕ 3.1. Технически удобно рассматривать не произвольные последовательности $\mu_{i} \rightarrow 0$, а последовательности, удовлетворяющие дополнительному условию:

(3.4) $1>\mu_{1}>\mu_{2}>\mu_{3}>\cdots$ и при некотором $q \in(0,1)$

$$
\limsup _{i \rightarrow \infty} \frac{\mu_{i+1}}{\mu_{i}} \geqslant q
$$

В [9] показано, что в этих предположениях из исходной последовательности можно выбрать подпоследовательность таким образом, чтобы (после соответствующей перенумерации) выполнялось условие $q^{2} \leqslant \mu_{i+1} / \mu_{i} \leqslant q$ при всех $i=1,2, \ldots$. Теперь для произвольной последовательности $\lambda_{i} \rightarrow 0$ обозначим $j(k)=\sup \{i: i=$ $\left.1,2, \ldots, \mu_{i}>\lambda_{k}\right\}$. Тогда $\mu_{j(k)}>\lambda_{k}$ и $\mu_{j(k)+1} \leqslant \lambda_{k}$, т.е. $\mu_{j(k)+1} \leqslant \lambda_{k}<\mu_{j(k)}$, $q^{2} \leqslant \lambda_{k} / \mu_{j(k)}<1$. Функция $\alpha_{k}(x)=\lambda_{k} \gamma\left(x / \lambda_{k}\right)$ связана с функцией $\beta_{k}(x)=$ $\mu_{j(k)} \gamma\left(x / \mu_{j(k)}\right)$ равенством $\alpha_{k}(x)=\nu_{k} \beta_{k}\left(x / \nu_{k}\right)$ с $\nu_{k}=\lambda_{k} / \mu_{j(k)} \in\left[q^{2}, 1\right]$. Отсюда сразу следует, что выполнение условия (3.3) для функций $\beta_{k}(x)$ влечет выполнение условия (3.3) для функций $\alpha_{k}(x)$. Тем самым выполнение условия (3.3) для какой-то одной последовательности $\mu_{i} \rightarrow 0$, удовлетворяющей условию $(3.4)$, влечет выполнение условия (3.3) в полном объеме (т.е. для произвольной последовательности $\left.\mu_{i} \rightarrow 0\right)$.

ЛЕМмА 3.1. Пусть выполнены условия (1.1), (1.2) и каждое из пространств $Z_{\lambda}, \lambda \in[c, d]$, удовлетворяет условиям (2.1) и (2.2). Пусть выполненьь условия (3.1)-(3.3) и

(3.5) функиия $h: \mathbb{R} \rightarrow[0, \infty)$ локально интегрируема и T-периодична,

(3.6) если функиия $z=(x, y)$ принадлежит $Z_{\lambda}, x(t)>0$ при всех $t \in \pi(z)$, то $y^{\prime}(t) \leqslant-\gamma(x(t))+h(t)$ почти при всех $t \in \pi(z)$.

Тогда

(3.7) если $z_{k}=\left(x_{k}, y_{k}\right) \in Z_{\lambda_{k}}, \sup \pi\left(z_{k}\right)-\inf \pi\left(z_{k}\right) \leqslant T, x_{k}\left(\inf \pi\left(z_{k}\right)\right)=$ $x_{k}\left(\sup \pi\left(z_{k}\right)\right)=0, x_{k}(t)>0$ npu $t \in\left(\inf \pi\left(z_{k}\right), \sup \pi\left(z_{k}\right)\right) u \sup x_{k}\left(\left[\inf \pi\left(z_{k}\right)\right.\right.$, $\left.\left.\sup \pi\left(z_{k}\right)\right]\right) \rightarrow \infty$ npu $k \rightarrow \infty$, mo $\lim _{k \rightarrow \infty}\left(\sup \pi\left(z_{k}\right)-\inf \pi\left(z_{k}\right)\right)=0$. 
ДокАЗАТЕЛЬСтво. В соответствии с (3.2) зафиксируем такие числа $Q_{0} \in \mathbb{R}$ и $\varepsilon>0$, что $\gamma(x)>\varepsilon^{2} x$ при $x \geqslant Q_{0}$. Возьмем произвольно $Q>Q_{0}$.

Мы находимся в предположениях лемм $\S 2$, и описанная там геометрия поведения решений распространяется на рассматриваемьй нами случай (леммы 2.2-2.5). Кроме того, $y_{k}\left(\inf \pi\left(z_{k}\right)\right) \rightarrow \infty$ (см. пп. 1) и 2) доказательства леммы 2.8).

Ввиду $T$-периодичности рассматриваемых пространств, не ограничивая общности, мы можем считать, что inf $\pi\left(z_{k}\right) \in[0, T]$. Тогда $\sup \pi\left(z_{k}\right) \leqslant 2 T$.

Начиная с некоторого $k$, траектория $z_{k}(t)$ заходит в область $x>Q$. Пусть $s_{k} \leqslant t_{k}$ - точки отрезка $\pi\left(z_{k}\right)$, соответствующие пересечению траекторией $z_{k}(t)$ вертикали $x=Q$ (т.е. $s_{k}$ соответствует точке входа, $t_{k}$ соответствует точке выхода).

В силу нашего выбора $Q$ при $s_{k} \leqslant t \leqslant t_{k}$ функция $y_{k}$ является убывающей, а функция $x_{k}$ сначала возрастает (до нуля $u_{k}$ функции $y_{k}$ ), а потом убывает.

Прежде чем начать общий анализ, выделим множество $P(Q)$ тех точек $t \in[0,2 T]$, для которых $h(t)>\varepsilon^{2} Q / 2$.

Пусть $\alpha>0$. Через $M_{k}$ обозначим множество тех точек $t \notin P(Q)$ отрезка $\left[s_{k}, t_{k}\right]$, для которых $\gamma\left(x_{k}(t)\right) \geqslant \alpha^{2} x_{k}(t)+\varepsilon^{2} Q / 2$. Так как при этом $h(t) \leqslant \varepsilon^{2} Q / 2$ и $-\gamma\left(x_{k}(t)\right) \leqslant-\alpha^{2} x_{k}(t)-\varepsilon^{2} Q / 2$, то $y_{k}^{\prime}(t) \leqslant-\gamma\left(x_{k}(t)\right)+h(t)<-\alpha^{2} x_{k}(t)$. Для функции $\varphi_{k}(t)=\operatorname{arctg}\left(-y_{k}(t) /\left(\alpha x_{k}(t)\right)\right), \varphi_{k}:\left[s_{k}, t_{k}\right] \rightarrow[-\pi / 2, \pi / 2]$, мы имеем

$$
\varphi_{k}^{\prime}(t)=\frac{\alpha\left(\left(-x_{k}(t)\right) y_{k}^{\prime}(t)+y_{k}^{2}(t)\right)}{\alpha^{2} x_{k}^{2}(t)+y_{k}^{2}(t)} .
$$

Если при этом еще $t \in M_{k}$, то

$$
\varphi_{k}^{\prime}(t) \geqslant \frac{\alpha\left(\alpha^{2} x_{k}^{2}(t)+y_{k}^{2}(t)\right)}{\alpha^{2} x_{k}^{2}(t)+y_{k}^{2}(t)}=\alpha .
$$

Мера образа $\varphi_{k}\left(M_{k}\right) \subseteq[-\pi / 2, \pi / 2]$ множества $M_{k}$ с одной стороны не больше $\pi$, а с другой стороны не меньше меры $\mu\left(M_{k}\right)$ множества $M_{k}$, умноженной на $\alpha$ : $\alpha \mu\left(M_{k}\right) \leqslant \mu\left(\varphi_{k}\left(M_{k}\right)\right) \leqslant \pi$. Поэтому

$$
\mu\left(M_{k}\right) \leqslant \frac{\pi}{\alpha}
$$

Для функции $\psi_{k}(t)=\operatorname{arctg}\left(-2 y_{k}(t) /\left(\varepsilon x_{k}(t)\right)\right)$ мы имеем

$$
\psi_{k}^{\prime}(t)=\frac{2 \varepsilon\left(\left(-x_{k}(t)\right) y_{k}^{\prime}(t)+2 y_{k}^{2}(t)\right)}{\varepsilon^{2} x_{k}^{2}(t)+4 y_{k}^{2}(t)} .
$$

Если $t \notin P(Q)$ (т.е. если $\left.h(t) \leqslant \varepsilon^{2} Q / 2\right)$, то $y_{k}^{\prime}(t)$ не превосходит $-\gamma\left(x_{k}(t)\right)+$ $h(t)<-\varepsilon^{2} x_{k}(t)+\varepsilon^{2} Q / 2=-\varepsilon^{2} x_{k}(t) / 2+\varepsilon^{2}\left(Q-x_{k}(t)\right) / 2$, а так как $x_{k}(t) \geqslant Q$, то $y_{k}^{\prime}(t) \leqslant-\varepsilon^{2} x_{k}(t) / 2$, и, следовательно, $\psi_{k}^{\prime}(t) \geqslant \varepsilon$. Если бы здесь не было точек $t \in$ $P(Q)$, то полученная оценка означала бы, что для любого числа $\delta>0$ траектория $z_{k}$ за время $2 \delta / \varepsilon$ проходит от луча $y=\delta x, x>0$ до положительного луча оси абсцисс, и за такое же время проходит далее до луча $y=-\delta x, x>0$. В общем случае

(3.9) мера $\mu\left(M_{k}^{\prime}(\delta)\right)$ множества $M_{k}^{\prime}(\delta)=\left\{t: t \in\left[s_{k}, t_{k}\right] \backslash P(Q),\left|y_{k}(t)\right| \leqslant \delta x_{k}(t)\right\}$ не больше $4 \delta / \varepsilon$.

Пусть $\mu_{k}=1 / x_{k}\left(u_{k}\right)$. Воспользуемся обозначениями (3.3). 


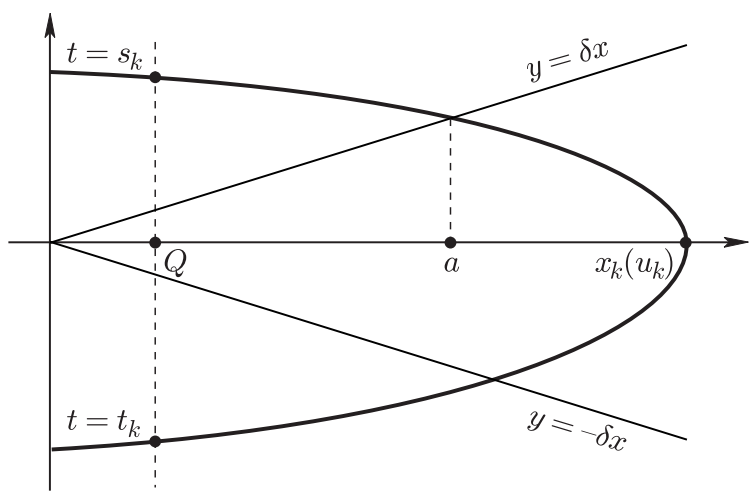

Рис. 7

Если точка $t \in\left[s_{k}, t_{k}\right]$ не принадлежит множеству $M_{k} \cup M_{k}^{\prime}(\delta) \cup P(Q)$, то она принадлежит либо множеству $N_{k}(\delta)=\left\{t: t \in\left[s_{k}, t_{k}\right] \backslash P(Q), y_{k}(t)>\delta x_{k}(t)\right.$, $\left.\gamma\left(x_{k}(t)\right)<\alpha^{2} x_{k}(t)+\varepsilon^{2} Q / 2\right\}$, либо множеству $N_{k}^{\prime}(\delta)=\left\{t: t \in\left[s_{k}, t_{k}\right] \backslash P(Q)\right.$, $\left.y_{k}(t)<-\delta x_{k}(t), \gamma\left(x_{k}(t)\right)<\alpha^{2} x_{k}(t)+\varepsilon^{2} Q / 2\right\}$.

Кривая пересекает луч $y=\delta x, x>0$ в некоторой точке $(a, \delta a)$ (рис. 7$)$. После этого $x_{k}^{\prime}(t)<\delta a$, поэтому

$$
\begin{gathered}
x_{k}\left(u_{k}\right)<a+\delta a\left(\frac{2 \delta}{\varepsilon}+\mu\left(P\left(Q_{0}\right)\right)\right)=a \frac{\varepsilon+2 \delta\left(\delta+\mu\left(P\left(Q_{0}\right)\right)\right)}{\varepsilon}, \\
a>\frac{\varepsilon x_{k}\left(u_{k}\right)}{\varepsilon+2 \delta\left(\delta+\mu\left(P\left(Q_{0}\right)\right)\right)} .
\end{gathered}
$$

При $t \in N_{k}(\delta)$ для функции $\xi_{k}(t)=\mu_{k} x_{k}(t), \xi_{k}:\left[s_{k}, t_{k}\right] \rightarrow[0,1]$, мы имеем $\xi_{k}^{\prime}(t)=\mu_{k} x_{k}^{\prime}(t) \geqslant \mu_{k} \delta a$, что больше не зависящего от $k$ числа $A=$ $\delta \varepsilon /\left(\varepsilon+2 \delta\left(\delta+\mu\left(P\left(Q_{0}\right)\right)\right)\right), g_{k}\left(\xi_{k}(t)\right)<\alpha^{2} \xi_{k}(t)+\varepsilon^{2} Q \mu_{k} / 2 \leqslant \alpha^{2} \xi_{k}(t)+\varepsilon^{2} Q / 2$. Поэтому мера $\mu\left(N_{k}(\delta)\right)$ множества $N_{k}(\delta)$ не больше меры $\mu\left(L_{k}\right)$ множества $L_{k}=\left\{v: v \in[0,1], g_{k}(v) \leqslant \alpha^{2}+\varepsilon^{2} Q / 2\right\}$, деленной на $A$. По аналогичным соображениям $\mu\left(N_{k}^{\prime}(\delta)\right) \leqslant \mu\left(L_{k}\right) / A$. Таким образом, мы имеем

$$
\begin{aligned}
\sup \pi\left(z_{k}\right)-\inf \pi\left(z_{k}\right) \leqslant & \left(s_{k}-\inf \pi\left(z_{k}\right)\right)+\left(\sup \pi\left(z_{k}\right)-t_{k}\right) \\
& +\mu\left(M_{k}\right)+\mu\left(M_{k}^{\prime}(\delta)\right)+\frac{2 \mu\left(L_{k}\right)}{A}+\mu(P(Q)) .
\end{aligned}
$$

В силу условия $(3.3) \mu\left(L_{k}\right) \rightarrow 0$. Из леммы 2.2 вытекает, что $\left(s_{k}-\inf \pi\left(z_{k}\right)\right) \rightarrow 0$ и $\left(\sup \pi\left(z_{k}\right)-t_{k}\right) \rightarrow 0$. Поэтому с учетом $(3.8)$ и $(3.9)$

$$
0 \leqslant \limsup _{k \rightarrow \infty}\left(\sup \pi\left(z_{k}\right)-\inf \pi\left(z_{k}\right)\right) \leqslant \frac{\pi}{\alpha}+\frac{4 \delta}{\varepsilon}+\mu(P(Q)) .
$$

Но числа $\alpha>0$ и $\delta>0$ взяты нами произвольно, поэтому

$$
0 \leqslant \limsup _{k \rightarrow \infty}\left(\sup \pi\left(z_{k}\right)-\inf \pi\left(z_{k}\right)\right) \leqslant \mu(P(Q))
$$

при любом $Q \geqslant Q_{0}$. В силу интегрируемости функции $h$ на отрезке $[0,2 T]$ мы имеем $\mu(P(Q)) \rightarrow 0$ при $Q \rightarrow \infty$. Поэтому из (3.10) вытекает равенство

$$
\lim _{k \rightarrow \infty}\left(\sup \pi\left(z_{k}\right)-\inf \pi\left(z_{k}\right)\right)=0 .
$$


Лемма доказана.

Таким образом, с ростом геометрических размеров траекторий время полуоборота стремится к нулю.

ЛЕМма 3.2. Пусть выполнень условия (1.1), (1.2) и каэсдое из пространств $Z_{\lambda}, \lambda \in[c, d]$, удовлетворяет условиям (2.1) и (2.2). Пусть $Q>0$, $l=1,2, \ldots$. Пусть выполнены условия (2.4), (3.7) и

$\left(3.7^{\prime}\right)$ ecлu $z_{k}=\left(x_{k}, y_{k}\right) \in Z_{\lambda_{k}}, \sup \pi\left(z_{k}\right)-\inf \pi\left(z_{k}\right) \leqslant T, x_{k}\left(\inf \pi\left(z_{k}\right)\right)=$ $x_{k}\left(\sup \pi\left(z_{k}\right)\right)=0, x_{k}(t)<0$ npu $t \in\left(\inf \pi\left(z_{k}\right), \sup \pi\left(z_{k}\right)\right) u \inf x_{k}\left(\left[\inf \pi\left(z_{k}\right)\right.\right.$, $\left.\left.\sup \pi\left(z_{k}\right)\right]\right) \rightarrow-\infty$ npu $k \rightarrow \infty$, mo $\lim _{k \rightarrow \infty}\left(\sup \pi\left(z_{k}\right)-\inf \pi\left(z_{k}\right)\right)=0$.

Тогда для любого $R>0$ найдется такое число $P(R, l)>R$, что если $z=(x, y) \in Z_{\lambda}^{+}, x(\inf \pi(z))=0, y(\inf \pi(z))>P(R, l)$, то кривая $z(t)$, $t \in[\inf \pi(z), \inf \pi(z)+T] \cap \pi(z)$, совершает не менее $l$ оборотов вокруг начала координат, пересекая положительный луч оси ординат в точках с ординатой больие или равной $R$.

ДокАЗАтЕльство. Допустив противное, получаем в силу леммы 2.7, что существуют такие функции $z_{m}=\left(x_{m}, y_{m}\right) \in Z_{\lambda_{m}}^{+}$и точки $t_{m} \in \pi\left(z_{m}\right) \cap(T, \infty)$, $m=1,2, \ldots$, что $x_{m}\left(\inf \pi\left(z_{m}\right)\right)=0$ и кривая $z_{m}(t), t \in\left[\inf \pi\left(z_{m}\right), t_{m}\right]$, совершает $l$ оборотов вокруг начала координат, на которых пересекает положительный луч оси ординат в точках с ординатой больше или равной $R+m$.

Из леммы 2.8 вытекает, что максимумы значений функции $x_{m}(t)$ в области $x>0$ стремятся к бесконечности, а минимумы значений функции $x_{m}(t)$ в области $x<0$ стремятся к $-\infty$. В силу условий $(3.7)$ и $\left(3.7^{\prime}\right)$ отсюда вытекает, что время, затрачиваемое кривой $z_{m}(t), t \in\left[\inf \pi\left(z_{m}\right), t_{m}\right]$, на прохождение областей $x<0$ и $x>0$, стремится к нулю. Но это означает, что, начиная с некоторого $m, l$-й оборот вокруг начала координат будет совершен кривой $z_{m}(t)$ также при $t<T$, что противоречит выбору функций $z_{m}$. Лемма доказана.

ЗАмЕчАнИЕ 3.2. В предположениях леммы 3.2 если функция $z=(x, y)$ принадлежит $Z_{\lambda}, \pi(z)=[0, T], z(0)=z(T)$, и кривая $z(t)$ пересекает положительный луч оси ординат в точках с ординатами больше или равными $R$ не более чем $l$ раз, то ординаты точек пересечения не превосходят $P(R, l+1)$.

ЛЕМмА 3.3. Пусть

(3.11) функции $\gamma_{\lambda}: \mathbb{R} \rightarrow \mathbb{R}, \lambda \in[c, d]$, локально интегрируемьи, функиия $G_{\lambda}(u)=\int_{0}^{u} \gamma_{\lambda}(s) d s$ непрерьвна по совокупности переменных $u, \lambda$, $\lim _{\lambda \in[c, d]} G_{\lambda}(u)=\infty$, функичя $h: \mathbb{R} \rightarrow[0, \infty)$ Т-периодична и интегрируема. $|u| \rightarrow \infty$

Тогда пространства решений включений

$$
x^{\prime \prime} \in\left[-\gamma_{\lambda}(x)-h(t),-\gamma_{\lambda}(x)+h(t)\right]
$$

удовлетворяют условию

(3.13) найдутся такие $R>0$ и $q \geqslant 1$, что если $z=(x, y)$ - решение одного из включений (3.12), $\sup \pi(z)-\inf \pi(z) \leqslant T$, точки $z(\inf \pi(z))$ и $z(\sup \pi(z))$ лежат на полохительном луче оси ординат и ордината точки $z(\inf \pi(z))$ больие $R$, mо

$$
\frac{1}{q} \leqslant \frac{y(\sup \pi(z))}{y(\inf \pi(z))} \leqslant q .
$$


ДоКАЗАТЕЛЬСТВО использует идею доказательства леммы 6.2 из [1]. Положим $V_{\lambda}(x, y)=G_{\lambda}(x)+y^{2} / 2, g_{0}=\min \left\{G_{\lambda}(u): u \in \mathbb{R}, \lambda \in[c, d]\right\}, R_{1}=1+$ $\sqrt{1-2 g_{0}}$. Так как $G_{\lambda}(x) \rightarrow \infty$ при $|x| \rightarrow \infty$ равномерно по $\lambda$, то найдется такое $R_{2}>0$, что $G_{\lambda}(x)>R_{1}$ при всех $\lambda \in[c, d]$ и всех $x$, для которых $|x|>R_{2}$. Пусть $R_{3}=\max \left\{V(x, y):|x| \leqslant R_{2},|y| \leqslant R_{1}\right\}$ и

$$
q=\sqrt{e^{\int_{0}^{T} h(t) d t}}
$$

Пусть $z=(x, y)$ - решение одного из включений $(3.12), s_{0}, t_{0} \in \pi(z), 0 \leqslant$ $t_{0}-s_{0} \leqslant T, \varphi(t)=V_{\lambda}(x(t), y(t))$ (с соответствуюшим $\lambda$ ) и $\varphi(t)>R_{3}$ при всех $t \in\left[s_{0}, t_{0}\right]$. Тогда при всех $t \in \pi(z)|x(t)|>R_{2}$ или $|y(t)|>R_{1}$. Если $|y(t)|>R_{1}$, то $(|y(t)|-1)^{2}>1-2 g_{0},|y(t)|^{2} / 2>|y(t)|-g_{0}$ и поэтому $|y(t)|<|y(t)|^{2} / 2+$ $g_{0} \leqslant \varphi(t)$. Если $|y(t)| \leqslant R_{1}$, то $|x(t)|>R_{2}, G_{\lambda}(x(t))>R_{1} \geqslant|y(t)|$ и поэтому $|y(t)| \leqslant G_{\lambda}(x(t))+|y(t)|^{2} / 2=\varphi(t)$. Так как

$$
\left|\varphi^{\prime}(t)\right|=\left|\gamma_{\lambda}(x(t)) x^{\prime}(t)+y(t) y^{\prime}(t)\right|=|y(t)|\left|x^{\prime \prime}(t)+\gamma_{\lambda}(x(t))\right|
$$

почти при всех $t \in \pi(z)$, то в силу сказанного $\left|\varphi^{\prime}(t)\right| \leqslant \varphi(t) h(t),\left|(\ln (\varphi(t)))^{\prime}\right| \leqslant h(t)$,

$$
\left|\ln \varphi\left(t_{0}\right)-\ln \varphi\left(s_{0}\right)\right| \leqslant \int_{0}^{T} h(t) d t, \quad \frac{1}{q^{2}} \leqslant \frac{\varphi\left(t_{0}\right)}{\varphi\left(s_{0}\right)} \leqslant q^{2} .
$$

Если теперь $z=(x, y)$ - решение одного из включений $(3.12), s_{0}, t_{0} \in \pi(z)$, $0 \leqslant t_{0}-s_{0} \leqslant T$ и $\varphi\left(s_{0}\right)>q^{2} R_{3}$, то в силу сказанного выше $\varphi(t)>R_{3}$ при всех $t \in\left[s_{0}, t_{0}\right]$.

Полагаем $R=q^{2} R_{3}$. Нам осталось только заметить, что если точки $z\left(s_{0}\right)$ и $z\left(t_{0}\right)$ лежат на оси ординат, то $\varphi\left(s_{0}\right)=\left(y\left(s_{0}\right)\right)^{2} / 2, \varphi\left(t_{0}\right)=\left(y\left(t_{0}\right)\right)^{2} / 2$ и поэтому $1 / q \leqslant\left|y\left(t_{0}\right) / y\left(s_{0}\right)\right| \leqslant q$. Лемма доказана.

В случае, когда отрезок $[c, d]$ состоит из одной точки, получаем

\section{СЛЕДСТВИЕ. Пусть}

$\left(3.11^{\prime}\right)$ функиия $\gamma: \mathbb{R} \rightarrow \mathbb{R}$ локально интегрируема, $G(u)=\int_{0}^{u} \gamma(s) d s$, $\lim _{|u| \rightarrow \infty} G(u)=\infty$, функиия $h: \mathbb{R} \rightarrow[0, \infty)$ T-периодична и интегрируема.

Тогда пространство решений включения

$$
x^{\prime \prime} \in[-\gamma(x)-h(t),-\gamma(x)+h(t)]
$$

удовлетворяет условию

$\left(3.13^{\prime}\right)$ найдутся такие $R>0$ и $q>1$, что если $z=(x, y)$ - решение включения $\left(3.12^{\prime}\right), \sup \pi(z)-\inf \pi(z) \leqslant T$, точки $z(\inf \pi(z))$ и $z(\sup \pi(z))$ лежст на положительном луче оси ординат и ордината точки $z(\inf \pi(z))$ больие $R$, mo

$$
\frac{1}{q} \leqslant \frac{y(\sup \pi(z))}{y(\inf \pi(z))} \leqslant q
$$

ЗАмЕчАнИЕ 3.3. Если пространство решений $Z$ лежит в пространстве решений $\left(3.12^{\prime}\right)$, то пространство $Z$ также удовлетворяет условию $\left(3.13^{\prime}\right)$. 
ЛЕмма 3.4. Пусть выполнень условия (1.1), (1.2), (3.11), каждое из пространств $Z_{\lambda}, \lambda \in[c, d]$, удовлетворяет условию (2.1) и лежст в пространстве решений включения (3.12). Пусть при $Q=p_{0}>0$ выполнены условия (2.3), (2.3'), (3.7) и (3.7'). Тогда выполнено условие (1.6), причем все точки, соответствующие постоянным решениям, принадлежсат одному из компактов в (1.6).

ДокАЗАТЕЛьство. По лемме 2.1 выполнено условие (2.2). Фиксируем $R>0$ и $q \geqslant 1$ в соответствии с леммой 3.3. При таком выборе если $z$ принадлежит $Z_{\lambda}, \sup \pi(z)-\inf \pi(z) \leqslant T, z(\inf \pi(z))=z(\sup \pi(z))$ и $z(t)=\overrightarrow{0}$ при некотором $t \in \pi(z)$, то ординаты точек пересечения кривой $z(t)$ с осью ординат ограничены сверху числом $R$.

Если теперь

(3.14) $z=(x, y) \in Z_{\lambda}, \sup \pi(z)-\inf \pi(z) \leqslant T, z(\inf \pi(z))=z(\sup \pi(z))$ и $x(t)=0, y(t)>R$ при некотором $t \in \pi(z)$,

то кривая $z(t)$ уже не может проходить через $\overrightarrow{0}$. Кривая не может лежать целиком в верхней полуплоскости, так как, находясь в верхней полуплоскости, текущая точка этой кривой должна двигаться вправо, что противоречило бы условию $z(\inf \pi(z))=z(\sup \pi(z))$. По аналогичным соображениям кривая не может лежать целиком в нижней полуплоскости. Пройти из верхней полуплоскости через нижнюю и вернуться обратно в верхнюю полуплоскость кривая может, лишь если обойдет начало координат $\overrightarrow{0}$ по часовой стрелке. Через $n(z)$ обозначим число совершаемых кривой $z(t)$ оборотов.

По лемме 2.8 множество $M$ функций $z$, принадлежащих $Z_{\lambda}, \lambda \in[c, d]$, таких, что $\sup \pi(z)-\inf \pi(z) \leqslant T, x(\inf \pi(z))=x(\sup \pi(z))=0, R \leqslant y(\inf \pi(z)) \leqslant q^{3} R$ и кривая $z(t)$ совершает 1 оборот вокруг начала координат, является ограниченным. Его замкнутость очевидна. Так как пространства $Z_{\lambda}$ принадлежат $R_{c}(U)$, то из сказанного вытекает компактность множества $M$. Так как непрерывная функция достигает на компакте своего минимума, то при некотором $\varepsilon>0$ время, затрачиваемое любой такой кривой $z(t)$ на 1 оборот вокруг начала координат, не меншше $\varepsilon$.

В силу выбора $q$ если $z=(x, y)$ принадлежит $Z_{\lambda}, \sup \pi(z)-\inf \pi(z) \leqslant T$, $z(\inf \pi(z))=z(\sup \pi(z))$ и $x(t)=0, q R \leqslant y(t) \leqslant q^{2} R$ при некотором $t \in \pi(z)$, то ординаты всех других пересечений этой кривой с осью ординат лежат в отрезке $\left[R, q^{3} R\right]$ и в силу сказанного вьше время, затрачиваемое кривой $z(t)$ на каждый оборот вокруг $\overrightarrow{0}$, не менее $\varepsilon$, поэтому $n(z) \leqslant T / \varepsilon$. Другими словами, если $z=(x, y) \in Z_{\lambda}, \sup \pi(z)-\inf \pi(z) \leqslant T, z(\inf \pi(z))=z(\sup \pi(z))$ и $n(z) \geqslant n_{0}$, где $n_{0}$ - любое целое число, большее $T / \varepsilon$, то кривая $z(t)$ не пересекает отрезок $\left[q R, q^{2} R\right]$ оси ординат и поэтому либо ординаты всех точек пересечения с осью ординат строго меньше $q R$, либо ординаты всех точек пересечения с осью ординат строго больше $q^{2} R$.

Для $n=1,2, \ldots$ через $D_{n}$ обозначим множество $\left\{(\lambda, z): \lambda \in[c, d], z \in Z_{\lambda}\right.$, $\pi(z)=[0, T], z(0)=z(T), n(z)=n$, ордината точки по крайней мере одного пересечения кривой $z(t)$ с осъю ординат больше $q R\}$. В силу замечания предыдущего абзаца при $n=n_{0}+1, n_{0}+2, \ldots$ если $(\lambda, z) \in D_{n}$, то ординаты всех точек пересечения кривой $z(t)$ с осью ординат больше $q^{2} R$.

Пусть $D_{0}=\left\{(\lambda, z): \lambda \in[c, d], z \in Z_{\lambda}, \pi(z)=[0, T], z(0)=z(T)\right.$, ординать точек пересечения кривой $z(t)$ с осъю ординат не больше $q R\}, D_{*}=D_{0} \cup D_{1} \cup$ $\cdots \cup D_{n_{0}}$. 
Из замечания 3.2 легко вытекает компактность множеств $D_{n}$. В силу сделанных замечаний компакты $D_{n}, n=n_{0}+1, n_{0}+2, \ldots$, попарно не пересекаются и не пересекаются с компактом $D_{*}$. Тем самым в силу представления $D=D_{*} \cup$ $D_{n_{0}+1} \cup D_{n_{0}+2} \cup \cdots$ мы убедились в выполнении условия $(1.6)$, и при этом только компакт $D_{*}$ содержит точки, соответствующие постоянным решениям. Лемма доказана.

СЛЕДСТВИЕ. Пусть выполнены условия (1.1), (1.2), (3.11'), каждое из пространств $Z_{\lambda}, \lambda \in[c, d]$, удовлетворяет условию (2.1) и лехсит в пространстве решений включения $\left(3.12^{\prime}\right)$. Пусть при $Q=p_{0}>0$ выполнены условия (2.3), $\left(2.3^{\prime}\right),(3.7)$ и $\left(3.7^{\prime}\right)$. Тогда выполнено условие (1.6).

ТЕОрема 3.1. Пусть выполнены условия (1.1), (2.1),

$\left(3.1^{\prime}\right)$ функиия $\gamma: \mathbb{R} \rightarrow \mathbb{R}$ непрерывна

$u$

$\left(3.2^{\prime}\right) \liminf _{|x| \rightarrow \infty} \frac{\gamma(x)}{x}>0$.

Пусть выполненьц условия (3.3) и

$\left(3.3^{\prime}\right)$ для любой последовательности $\mu_{i} \rightarrow 0, \mu_{i}>0$, последовательность функиий $g_{i}(x)=\mu_{i} \gamma\left(x / \mu_{i}\right)$ сходится на отрезке $[-1,0] \kappa-\infty$ в смысле выполнения следующего условия: для любого $R>0$ мера мнохества $\{x: x \in[-1,0]$, $\left.g_{i}(x)>-R\right\}$ стремится $к$ нулю.

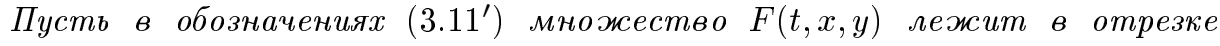
$[-\gamma(x)-h(t),-\gamma(x)+h(t)]$ при всех $(t, x, y) \in U$. Тогда существует такое решение $x(t)$ включения $x^{\prime \prime} \in F\left(t, x, x^{\prime}\right)$, что $\pi(x)=[0, T], x(0)=x(T)$ u $x^{\prime}(0)=x^{\prime}(T)$.

ДокАЗАтЕльство. 1) Ввиду $\left(3.2^{\prime}\right)$ найдутся такие $Q>0, \varepsilon>0$, что $\gamma(x) / x>\varepsilon^{2}$ при $|x| \geqslant Q$. При этом выберем $\varepsilon$ так, чтобы линейное уравнение $x^{\prime \prime}+\varepsilon^{2} x=0$ не имело ненулевых $T$-периодических решений.

2) Через $Y$ обозначим пространство решений уравнения $x^{\prime \prime}+x^{\prime} /\left(1+\left|x^{\prime}\right|\right)+$ $\gamma(x)=0$. Уравнение взято из $[1 ; \S 6.1]$.

Рассмотрим пространства $Z_{\lambda}, \lambda \in[0, T]$, определив их в слое $[0, T] \times \mathbb{R}^{2}$ как $Z *_{\lambda} Y$ и затем доопределяя их во всем пространстве $\mathbb{R} \times \mathbb{R}^{2}$ по $T$-периодичности.

Здесь $Z *_{s} Y$ обозначает результат подклеивания пространства $Y$ к пространству $Z$, причем в качестве вспомогательной функции $h$ берется проектирование произведения $\mathbb{R} \times \mathbb{R}^{n}$ на сомножитель $\mathbb{R}$, т.е. пространство $Z *_{s} Y$ при $t \leqslant s$ совпадает с пространством $Z$, а при $t \geqslant s-$ совпадает с пространством $Y$ (см. [6; $\S$ VIII.7] или $[8 ; \S 11.5])$.

3) Для $\lambda \in[-1,0]$ через $Z_{\lambda}\left(\in R_{c e h}(U)\right)[9]$ обозначим пространство решений уравнения $x^{\prime \prime}-\lambda \varepsilon^{2} x+(1+\lambda)\left(x^{\prime} /\left(1+\left|x^{\prime}\right|\right)+\gamma(x)\right)=0$.

4) Пространство $Z_{0}$ мы определили дважды. Но оба способа определения дают нам один и тот же результат. В обоих случаях пространство $Z_{0}$ совпадает с пространством $Y$ решений уравнения $x^{\prime \prime}+x^{\prime} /\left(1+\left|x^{\prime}\right|\right)+\gamma(x)=0$, склеивая, таким образом, гомотопии в 2) и 3 ). Свойства этой склеенной гомотопии Г, при которой параметр $\lambda$ сначала меняется от -1 до 0 , а потом от 0 до $T$, мы будем обсуждать.

$5)$ Не ограничивая общности, мы можем считать, что $h(t) \geqslant 1$ при всех $t$. Тогда все пространства $Z_{\lambda}$ при $\lambda \in[0, T]$ лежат в пространстве решений включения $\left(3.11^{\prime}\right)$. Поэтому к гомотопии $Z_{\lambda}, \lambda \in[0, T]$, применимо следствие леммы 3.4 . 
Но если $x(t)$ - решение уравнения

$$
x^{\prime \prime}-\lambda \varepsilon^{2} x+(1+\lambda)\left(\frac{x^{\prime}}{1+\left|x^{\prime}\right|}+\gamma(x)\right)=0, \quad \lambda \in(-1,0],
$$

TO

$$
x^{\prime \prime}(t) x^{\prime}(t)-\lambda \varepsilon^{2} x(t) x^{\prime}(t)+(1+\lambda)\left(\frac{\left(x^{\prime}(t)\right)^{2}}{1+\left|x^{\prime}(t)\right|}+\gamma(x(t)) x^{\prime}(t)\right)=0 .
$$

Если при этом $\pi(x)=[0, T], x(0)=x(T)$ и $x^{\prime}(0)=x^{\prime}(T)$, то интегрируя по $t$ от 0 до $T$, получаем

$$
(1+\lambda) \int_{0}^{T} \frac{\left(x^{\prime}(t)\right)^{2}}{1+\left|x^{\prime}(t)\right|} d t=0,
$$

что возможно лишь если функция $x(t)$ постоянна: $x(t) \equiv \xi$. Тогда $-\lambda \varepsilon^{2} \xi+$ $(1+\lambda) \gamma(\xi)=0$. Но если $|\xi| \geqslant Q$, то $-\lambda \varepsilon^{2} \xi+(1+\lambda) \gamma(\xi)>-\lambda \varepsilon^{2} \xi+$ $(1+\lambda) \varepsilon^{2}|\xi|=\lambda \varepsilon^{2}(|\xi|-\xi)+\varepsilon^{2}|\xi| \geqslant \varepsilon^{2}|\xi|>0$. Таким образом, $|\xi|<Q$. Поэтому если мы отнесем соответствующие точки к компакту леммы 3.4 , содержашему точки, соответствующие постоянным решениям, то выполнение требуемого в (1.6) условия сохраняется.

В силу нашего выбора $\varepsilon$ при $\lambda=-1$ есть единственное $T$-периодическое решение $x(t) \equiv 0$.

Таким образом, условие (1.6) выполнено для всей гомотопии $Г$.

При $\lambda=-1$ в силу линейности уравнения сдвиг линеен. В силу нашего выбора $\varepsilon$ сдвиг невырожден. Поэтому его степень равна \pm 1 на окружности любого радиуса $r>0$. Таким образом, вьполнены все предположения формулировки теоремы 1.1 , если в качестве пространства $Z_{c}$ теоремы 1.1 взять пространство $Z_{-1}$ решений уравнения $x^{\prime \prime}+\varepsilon^{2} x=0$. Ссылка на теорему 1.1 завершает рассуждение. Теорема доказана.

В [1] приведена следующая

ТЕОРЕМА 3.2. Пусть непрерьвная функиия $f: \mathbb{R} \rightarrow \mathbb{R}$ удовлетворяет условию

$$
\lim _{|x| \rightarrow \infty} \frac{f(x)}{x}=\infty
$$

непрерывная функция $e: \mathbb{R} \rightarrow \mathbb{R} T$-периодична. Тогда существует такое решение $x(t)$ уравнения $x^{\prime \prime}+f(x(t))=e(t)$, что $\pi(x)=[0, T], x(0)=x(T) u$ $x^{\prime}(0)=x^{\prime}(T)$.

ДокАЗАТЕльство в нашем случае сводится к ссылке на теорему 3.1. При этом надо положить $\gamma=f$ и $h(x) \equiv \sup \{|e(t)|: t \in \mathbb{R}\}$. Выполнение условий $\left(3.1^{\prime}\right)-\left(3.3^{\prime}\right)$ и (3.3) вытекает из (3.15). Теорема доказана.

ПримеР 3.1. Пусть выполнены предположения теоремы 3.2. Тогда существует такое решение $x(t)$ уравнения

$$
x^{\prime \prime}+f(x)\left(\sin ^{2}(\ln |x|)\right)^{1 /|x|}+x=e(t),
$$

что $\pi(x)=[0, T], x(0)=x(T)$ и $x^{\prime}(0)=x^{\prime}(T)$. Полагаем

$$
\gamma(x)=f(x)\left(\sin ^{2}(\ln |x|)\right)^{1 /|x|}+x, \quad h \equiv \sup \{|e(t)|: t \in \mathbb{R}\}, \quad \lambda_{i}=(2 \pi i)^{-1} .
$$


Тогда $g_{i}(x)=\lambda_{i} \gamma\left(x / \lambda_{i}\right)=\lambda_{i} \gamma\left(x / \lambda_{i}\right)\left(\sin ^{2}(\ln |x|)\right)^{\lambda_{i} /|x|}+x$. Неотрицательньй множитель $A=\left(\sin ^{2}(\ln |x|)\right)^{\lambda_{i} /|x|}$ обрашается в нуль в точках $x= \pm e^{ \pm \pi i}$, поэтому

$$
\liminf _{|x| \rightarrow \infty} \frac{\gamma(x)}{x}=1
$$

На любом отрезке, не задевающем эти точки, множитель $A$ равномерно сходится к единице. В силу этого, леммы 3.1 и (3.15) мы имеем выполнение условий (3.3) и $\left(3.3^{\prime}\right)$. В силу теоремы 3.1 сушествует такое решение $x(t)$ уравнения

$$
x^{\prime \prime}+f(x)\left(\sin ^{2}(\ln |x|)\right)^{1 /|x|}+x=e(t)
$$

что $\pi(x)=[0, T], x(0)=x(T)$ и $x^{\prime}(0)=x^{\prime}(T)$.

\section{§4. Смешанные условия на рост слагаемых}

Кажется, еще никто не обратил внимание, что условия типа (3.15) могут фигурировать в списке условий спектра Фучика. В этом параграфе мы сопоставим его в виде (3.1)-(3.3) с наработанной идеологией спектра Фучика [9], [13]-[15]. Для упрощения обозначений будем рассматривать конкретный временной отрезок $[0, \pi]$.

В предварительных рассуждениях при описании поведения решений в левой полуплоскости $x<0$ будем ориентироваться на условие $\left(3.7^{\prime}\right)$. Сформулируем соответствуюшее ограничение для правой полуплоскости $x \geqslant 0$. Пусть $U_{+}=$ $(-\infty, \infty) \times([0, \infty) \times(-\infty, \infty))$ и

(4.1) каждое из пространств $Z_{i} \in P_{c e h}^{\pi}(U), i=1,2 \ldots$, имеет вид $(2.1)$, последовательность пространств $Z_{i}$ при $i \rightarrow \infty$ сходится на множестве $U_{+}$к пространству решений включения $x^{\prime \prime} \in-\left[m^{2},(m+1)^{2}\right] x, m=1,2, \ldots$, и задача $z=(x, y) \in \lim \operatorname{top} \sup _{i \rightarrow \infty} Z_{i}, \sup \pi(z)-\inf \pi(z)=\pi / m$ или $\sup \pi(z)-$ $\inf \pi(z)=\pi /(m+1), x(t)>0$ при $t \in\langle\pi(z)\rangle, x(\inf \pi(z))=x(\sup \pi(z))=0$, $y(\inf \pi(z))>0$ не имеет решения.

ЛЕмма 4.1. В обозначениях (1.1), (4.1) для любъх $0<R_{0} \leqslant R_{1}<\infty$ найдется такое $\varepsilon>0$, что если $z_{i}=\left(x_{i}, y_{i}\right) \in Z_{i}, i=1,2, \ldots, x_{i}(t)>0$ npu $t \in\left\langle\pi\left(z_{i}\right)\right\rangle, x_{i}\left(\inf \pi\left(z_{i}\right)\right)=x_{i}\left(\sup \pi\left(z_{i}\right)\right)=0, y_{i}\left(\inf \pi\left(z_{i}\right)\right) \in\left[R_{0}, R_{1}\right], m o$, начиная с некоторого $i=i_{0}, \pi /(m+1)+\varepsilon \leqslant \sup \pi\left(z_{i}\right)-\inf \pi\left(z_{i}\right) \leqslant \pi / m-\varepsilon$.

ДоказАтельство. Строение пространств $Z_{i}$ при $x \leqslant 0$ не отражается на обсуждаемых связях. Поэтому мы можем предположить дополнительно, что пространство $Z_{i}$ симметрично относительно $\overrightarrow{0}$, т.е. не меняется при замене переменных $(t, x, y) \mapsto(t,-x,-y)$.

В силу $\pi$-периодичности рассматриваемых пространств без ограничения общности мы можем предполагать, что $0 \leqslant \inf \pi\left(z_{m}\right) \leqslant \pi$.

Допустив, что доказываемое не имеет места, получаем существование последовательности $\left\{z_{i}=\left(x_{i}, y_{i}\right): i \in A\right\}$, для всех членов которой $z_{i}$ принадлежит $Z_{i}$, $\inf \pi\left(z_{i}\right) \in[0, \pi], \sup \pi\left(z_{i}\right)-\inf \pi\left(z_{i}\right) \leqslant \pi /(m+1)+1 / i, x_{i}(t)>0$ при $t \in\left\langle\pi\left(z_{i}\right)\right\rangle$, $x_{i}\left(\inf \pi\left(z_{i}\right)\right)=x_{i}\left(\sup \pi\left(z_{i}\right)\right)=0, y_{i}\left(\inf \pi\left(z_{i}\right)\right) \in\left[R_{0}, R_{1}\right]$ либо для всех членов $z_{i} \in Z_{i}, \inf \pi\left(z_{i}\right) \in[0, \pi], \sup \pi\left(z_{i}\right)-\inf \pi\left(z_{i}\right) \geqslant \pi / m-1 / i, x_{i}(t)>0$ при $t \in\left\langle\pi\left(z_{i}\right)\right\rangle$, $x_{i}\left(\inf \pi\left(z_{i}\right)\right)=x_{i}\left(\sup \pi\left(z_{i}\right)\right)=0, y_{i}\left(\inf \pi\left(z_{i}\right)\right) \in\left[R_{0}, R_{1}\right]$. Случаи вполне аналогичны. Ограничимся рассмотрением первого. 
Каждую из функций $z_{i}$ максимально продолжим вправо до функции $z_{i}^{*} \in Z_{i}^{+}$. По лемме 10.4.1r из [8] существуют такое максимально продолженное вправо решение $z=\left(x, x^{\prime}\right)$ включения $x^{\prime \prime} \in-\left[m^{2},(m+1)^{2}\right] x$ и такая подпоследовательность $\left\{z_{i}^{*}: i \in A_{0}\right\}$ последовательности $\left\{z_{i}^{*}: i \in A\right\}$, что для любой точки $t \in \pi(z)$ справедливо следующее: $\pi\left(z_{i}\right) \ni t$, начиная с некоторого $i=i_{0}$, и последовательность $\left\{\left.z_{i}^{*}\right|_{\left[\inf \pi\left(z_{i}\right), t\right]}: i \in A\right\}$ сходится к функции $\left.z\right|_{(-\infty, t] \cap \pi(z)}$.

Но пространство решений включения $x^{\prime \prime} \in-\left[m^{2},(m+1)^{2}\right] x$ конично. В силу результатов $[16 ; \S 1]$ ни одно решение включения $x^{\prime \prime} \in-\left[m^{2},(m+1)^{2}\right] x$ не может уйти за конечное время на бесконечность, поэтому inf $\pi(z)+\pi /(m+1) \in \pi(z)$.

При этом так как $x^{\prime}(\inf \pi(z)) \geqslant R_{0}>0$, то $x(t)>0$ при $t \in\left(\inf \pi(z), t_{0}\right)$ для некоторого $t_{0}>\inf \pi(z)$. Кроме того ввиду аналогичного свойства функций $x_{i}$ на отрезке $\left[t_{0}, \inf \pi(z)+\pi /(m+1)\right]$ функция $x$ должна обратиться в нуль. Но среди решений включения $x^{\prime \prime} \in-\left[m^{2},(m+1)^{2}\right] x$ этому условию удовлетворяют только функции вида $a \cos \left((m+1) t+t_{0}\right)$ и, следовательно, $x(t)>0$ при $t \in(\inf \pi(z), \inf \pi(z)+\pi /(m+1)), x(\inf \pi(z))=x(\inf \pi(z)+\pi /(m+1))=0$. Но существование такой функции $z$ противоречит условию (4.1). Сделанное допушение привело нас к противоречию. Лемма доказана.

ЛЕмма 4.2. Пусть в обозначениях (1.1), (1.2)

(4.2) пространство $Y_{\lambda, \mu}$ получается из пространства $Z_{\lambda}$ при замене переменных $(t, x) \mapsto(t, \mu x)$,

$0<R_{0} \leqslant R_{1}<\infty u$

(4.3) для некоторой последовательности $\mu_{i} \rightarrow 0$ при любъх $\lambda_{i} \in[c, d]$ последовательность пространств $Y_{\lambda_{i}, \mu_{i}}$ сходится на мнохсестве $U_{+} \kappa$ пространству решений включения $x^{\prime \prime} \in-\left[m^{2},(m+1)^{2}\right] x, m=1,2, \ldots$, и задача $z=(x, y) \in \operatorname{limtop} \sup _{i \rightarrow \infty} Z_{\lambda_{i}, \mu_{i}}, \sup \pi(z)-\inf \pi(z)=\pi / m$ uлu $\sup \pi(z)-$ $\inf \pi(z)=\pi /(m+1), x(t)>0$ npu $t \in\langle\pi(z)\rangle, x(\inf \pi(z))=x(\sup \pi(z))=0$, $y(\inf \pi(z))>0$ не имеет решения.

Тогда найдутся такие $i_{0}=1,2, \ldots, \varepsilon>0$, что если $z=(x, y) \in Y_{\lambda, \mu_{i}}, i=$ $i_{0}, i_{0}+1, i_{0}+2, \ldots, \lambda \in[c, d], x(t)>0$ npu $t \in\langle\pi(z)\rangle, x(\inf \pi(z))=x(\sup \pi(z))=0$, $y(\inf \pi(z)) \in\left[R_{0}, R_{1}\right]$, mo $\pi /(m+1)+\varepsilon \leqslant \sup \pi(z)-\inf \pi(z) \leqslant \pi / m-\varepsilon$.

ДокАЗАтЕльство. Допустив противное, получаем существование такой последовательности функций $z_{i}=\left(x_{i}, y_{i}\right) \in Y_{\lambda_{i}, \mu_{i}}, i \in A$, что $x_{i}(t)>0$ при $t \in\left\langle\pi\left(z_{i}\right)\right\rangle, x_{i}\left(\inf \pi\left(z_{i}\right)\right)=x_{i}\left(\sup \pi\left(z_{i}\right)\right)=0, y(\inf \pi(z)) \in\left[R_{0}, R_{1}\right]$,

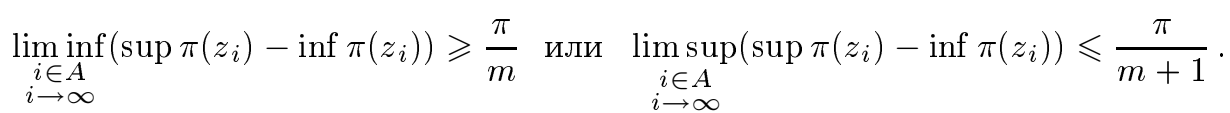

В силу (4.3) это входит в противоречие с доказанным в лемме 4.1. Лемма доказана.

ЛЕмма 4.3. Пусть в обозначениях (1.1), (1.2), (4.2) и (3.11) каждое из пространств $Z_{\lambda}, \lambda \in[c, d]$, лежит в пространстве решений включения $x^{\prime \prime} \in\left[-\gamma_{\lambda}(x)-h(t),-\gamma_{\lambda}(x)+h(t)\right]$ и выполнены условия (3.7') и (4.3). Тогда найдется такое $i_{0}=1,2, \ldots$, что если $z=(x, y) \in Y_{\lambda, \mu_{i}}, i=i_{0}, i_{0}+1, i_{0}+2, \ldots$, $\lambda \in[c, d], \pi(z)=[0, \pi]$ и $z(0)=z(\pi)$, то ординаты точек пересечения кривой $z(t)$ с осъю ординат отличньц от единиць. 
ДоКАЗАТЕЛЬСтво. В силу леммы 3.3 найдется $q \geqslant 1$, для которого выполнено условие (3.13). Положим $R_{0}=1 / q$ и $R_{1}=q$.

В силу условия $\left(3.7^{\prime}\right)$ и следствия леммы 2.3 найдется такое число $R_{2}>0$, что если $z=(x, y) \in Z_{\lambda}, \pi(z)=\left[s_{0}, t_{0}\right], x\left(s_{0}\right)=x\left(t_{0}\right)=0, x(t)<0$ при $t \in\left(s_{0}, t_{0}\right)$ и $y\left(s_{0}\right)<-R_{2}$, то $t_{0}-s_{0}<\varepsilon$.

В силу лемм 2.5 и 2.7 найдется такое число $R_{3}>0$, что если $z=(x, y)$ принадлежит $Z_{\lambda}^{+}, x(\inf \pi(z))=0, y(\inf \pi(z))>R_{3}$, то при некотором $t_{0} \in \pi(z)$ кривая $z(t), t \in\left[\inf \pi(z), t_{0}\right]$, совершает $m+1$ оборот вокруг начала координат, пересекая отрицательный луч оси ординат в точках с ординатой менњше $-R_{2}$.

В соответствии с леммой 4.2 зафиксируем $i_{0}$ так, что $1 / \mu_{i_{0}}>R_{3}$. В силу леммы 3.3 если кривая $z(t), z \in Y_{\lambda, \mu_{i}}$ с $i \geqslant i_{0}, \pi(z) \subseteq[0, \pi]$, пересекает ось ординат в точке с ординатой 1 , то ординаты других возможных ее пересечений с осью ординат лежат в отрезке $\left[R_{0}, R_{1}\right]$. Поэтому на один оборот кривая $z(t)$ затрачивает время, строго больше, чем $\pi /(m+1)$, и строго меньше, чем $\pi / m$. Поэтому на не более чем $m$ оборотов кривая затрачивает время меншше, чем $\pi$, а на не менее чем $m+1$ оборот - больше, чем $\pi$.

Это означает, что нет таких функций $z=(x, y) \in Y_{\lambda, \mu_{i}}$, что $\pi(z)=[0, \pi]$, $x(0)=x(\pi)$, и кривая $z(t)$ по крайней мере один раз пересекает положительньй луч оси ординат в точке с ординатой 1 . Лемма доказана.

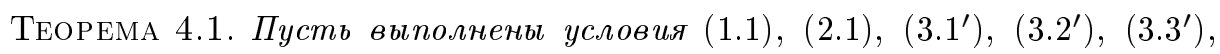
$\left(3.11^{\prime}\right)$ u $F(t, x, y) \subseteq[-\gamma(x)+h(t),-\gamma(x)-h(t)]$ npu $(t, x, y) \in U$. Пусть

$$
m^{2} \leqslant \liminf _{x \rightarrow \infty} \frac{\gamma(x)}{x} \leqslant \limsup _{x \rightarrow \infty} \frac{\gamma(x)}{x} \leqslant(m+1)^{2}, \quad m=1,2, \ldots
$$

$u$

$$
m^{2}<\limsup _{x \rightarrow \infty} \frac{1}{2 x^{2}} \int_{0}^{x} \gamma(u) d u, \quad \liminf _{x \rightarrow \infty} \frac{1}{2 x^{2}} \int_{0}^{x} \gamma(u) d u<(m+1)^{2}
$$

Тогда существует такое решение $x(t)$ включения $x^{\prime \prime} \in F\left(t, x, x^{\prime}\right)$, что $\pi(x)=$ $[0, \pi], x(0)=x(\pi) u x^{\prime}(0)=x^{\prime}(\pi)$.

ДокАЗАТЕльство. 1) Из (4.5) следует существование такой последовательности ${ }^{2} x_{i} \rightarrow \infty$, что предел

$$
\xi=\lim _{i \rightarrow \infty} \frac{1}{2 x_{i}^{2}} \int_{0}^{x_{i}} \gamma(u) d u
$$

существует и $m^{2}<\xi<(m+1)^{2}$. Положим $\mu_{i}=1 / x_{i}$.

Ввиду $\left(3.2^{\prime}\right)$ найдутся такие $Q>0$ и $\varepsilon>0$, что $\gamma(x) / x>\varepsilon^{2}$ при $|x| \geqslant Q$. При этом выберем $\varepsilon$ так, чтобы линейное уравнение $x^{\prime \prime}+\varepsilon^{2} x=0$ не имело ненулевых $T$-периодических решений.

2) Через $Y$ обозначим пространство решений уравнения $x^{\prime \prime}+x^{\prime} /\left(1+\left|x^{\prime}\right|\right)+$ $\gamma(x)=0$.

Рассмотрим пространства $Z_{\lambda}, \lambda \in[0, \pi]$, определив их в слое $[0, \pi] \times \mathbb{R}^{2}$ как $Z *_{\lambda} Y$ и затем доопределяя их во всем пространстве $\mathbb{R} \times \mathbb{R}^{2}$ по $\pi$-периодичности ${ }^{3}$.

\footnotetext{
${ }^{2}$ В контексте рассматриваемых вопросов - наблюдение С. Р. Габдрахманова.

${ }^{3}$ Обозначение $Z *_{s} Y$ было введено при доказательстве теоремы 3.1 .
} 
3) Для $\lambda \in[-1,0]$ через $Z_{\lambda}\left(\in R_{c e h}(U)\right)[9]$ обозначим пространство решений уравнения

$$
x^{\prime \prime}-\lambda \varepsilon^{2} x+(1+\lambda)\left(\frac{x^{\prime}}{1+\left|x^{\prime}\right|}+\gamma(x)\right)=0 .
$$

4) Пространство $Z_{0}$ мы определили дважды. Но оба способа определения дают нам один и тот же результат. В обоих случаях пространство $Z_{0}$ совпадает с пространством $Y$ решений уравнения $x^{\prime \prime}+x^{\prime} /\left(1+\left|x^{\prime}\right|\right)+\gamma(x)=0$, склеивая, таким образом, гомотопии в 2) и 3 ). Свойства этой склеенной гомотопии $Г$, при которой параметр $\lambda$ сначала меняется от -1 до 0 , а потом от 0 до $\pi$, мы будем обсуждать.

$5)$ В силу леммы 2.1 при любом $\lambda \in[-1, \pi]$ пространство $Z_{\lambda}$ удовлетворяет условию (2.2).

6) Определим пространство $Y_{\lambda, \mu}$ в соответствии с (4.2).

Не ограничивая общности, мы можем считать, что $h(t) \geqslant 1$ при всех $t$. Тогда все пространства $Z_{\lambda}$ при $\lambda \in[0, \pi]$ лежат в пространстве решений включения $\left(3.11^{\prime}\right)$.

При $\lambda \in[-1,0]$ в качестве пространства $Z_{\lambda}$ мы имеем пространство решений уравнения

$$
x^{\prime \prime}+\frac{(1+\lambda) x^{\prime}}{1+\left|x^{\prime}\right|}+\gamma_{\lambda}(x)=0
$$

$\mathrm{c} \gamma_{\lambda}(x)=(1+\lambda) \gamma(x)-\lambda \varepsilon^{2} x$.

Для $\lambda \in[0, \pi]$ условие (4.4) влечет выполнение первой части условия (4.3) (о сходимости к пространству решений включения $\left.x^{\prime \prime} \in-\left[m^{2},(m+1)^{2}\right] x\right)$, а наш выбор последовательности $\mu_{i} \rightarrow 0$ влечет выполнение второй части части условия (4.3) (об отсутствии в предельном пространстве соответствующих решений [13]-[15]). Таким образом, мы имеем возможность сослаться на лемму 4.3. Учитывая связь между $Z_{\lambda}$ и $Y_{\lambda, \mu_{i}}$, это означает, что найдется такое $R>0,{ }^{4}$ что если $z=(x, y) \in Z_{\lambda}, \lambda \in[0, \pi], \pi(z)=[0, \pi]$ и $z(0)=z(\pi)$, то ординаты точек пересечения кривой $z(t)$ с осью ординат отличны от $R$.

Как и в доказательстве теоремы 3.1 , при $\lambda \in[-1,0]$ периодические решения являются константами. Поэтому полученная в предыдущем параграфе оценка продолжается и на эти значения параметра $\lambda$.

По лемме 2.8 множество $M$ функций $z=(x, y) \in Z_{\lambda}, \lambda \in[c, d], \pi(z)=[0, \pi]$ и $z(0)=z(\pi)$, для которых ординаты точек пересечения кривой $z(t)$ с положительным лучом оси ординат не превосходят $R$, является ограниченньм.

Через $C$ обозначим верхнюю грань значений $\sup \{|x(t)|,|y(t)|: t \in \pi(z)\}$ по всем решениям $z \in M$.

Через $W$ обозначим множество функций $z=(x, y)$ пространства $C\left([0, \pi], \mathbb{R}^{2}\right)$ таких, что $\sup \{|x(t)|,|y(t)|: t \in[0, \pi]\}<2 C$ и ординаты точек пересечения кривой $z(t)$ с положительным лучом оси ординат меньше $R$. Множество $W$ открыто и ограничено в пространстве $C_{s}^{+}(U, 0)$.

При таком выборе если функция $z=(x, y)$ принадлежит $[W] \backslash W, \pi(z)=[0, \pi]$ и $z(0)=z(\pi)$, то ординаты точек пересечения кривой $z(t)$ с положительным лучом оси ординат не превосходят $R$. Поэтому в силу определения $C$ верхняя грань $\sup \{|x(t)|,|y(t)|: t \in[0, \pi]\}$ не превосходит $C<2 C$. Но так как при этом $z \notin W$, то ордината хотя бы одной из точек пересечения кривой $z(t)$ с положительным лучом оси ординат равна $R$. Существование такой функции $z$ противоречит выбору числа $R$. Таким образом, множество $W$ удовлетворяет условиям $(1.8),(1.9)$. При

\footnotetext{
${ }^{4}$ А именно $R=1 / \mu_{i_{0}}=x_{i_{0}}$.
} 
$\lambda=-1$ мы имеем линейное уравнение $x^{\prime \prime}+\varepsilon^{2} x=0$, поэтому выполнено и условие (1.10) с $c=-1$. Нам остается сослаться на теорему 1.2. Теорема доказана.

ТЕОРема 4.2. Пусть непрерывная функиия $\gamma: \mathbb{R} \rightarrow \mathbb{R}$ удовлетворяет условиям (4.4) и (4.5). Пусть непрерьвная функиия $е: \mathbb{R} \rightarrow \mathbb{R} \pi$-периодична. Тогда существует такое решение $x(t)$ уравнения $x^{\prime \prime}+\gamma(x)=e(t)$, что $\pi(x)=[0, \pi]$, $x(0)=x(\pi)$ u $x^{\prime}(0)=x^{\prime}(\pi)$.

ДоКАЗАТЕЛЬСТво сводится к ссылке на теорему 4.1. При этом надо положить $h(t) \equiv \sup \{|e(t)|: t \in \mathbb{R}\}$. Теорема доказана.

Естественно, условия теорем 4.1 и 4.2 допускают “шевеления" типа приведенных в примере 3.1 .

\section{Список литературы}

1. Mawhin J. Continuation theorems and periodic solutions of ordinary differential equations // Topological methods in differential equations and inclusions. Dordrecht: Kluwer Acad. Publ., 1995. P. 291-376.

2. Филиппов В.В. О гомологических свойствах множеств решений обыкновенных дифференциальных уравнений // Матем. сб. 1997. Т. 188. №6. С. 139-160.

3. Филиппов В. В. Смесь методов Лерэ-Шаудера и Пуанкаре-Андронова в задаче о периодических решениях обькновенных дифференциальных уравнений // Дифференц. уравнения. 1999. Т. 35. № 12. С. 1709-1711.

4. Filippov V. V. Remarks on periodic solutions of ordinary differential equations // J. Dynam. Control Systems. 2000. V. 6. № 3. P. 431-451.

5. Филиппов $B$. В. Топологическое строение пространств решений обыкновенных дифференциальных уравнений // УМН. 1993. Т. 48. № 1. С. 103-154.

6. Филиппов В.В. Пространства решений обыкновенных дифференциальных уравнений. М.: Изд-во МГУ, 1993.

7. Filippov $V$. V. Basic topological structures of the theory of ordinary differential equations // Topology in nonlinear analysis. Warsawa: Polish Acad. Sci., 1996. P. 171-192. (Banach Center Publ. V. 35.)

8. Filippov $V$. V. Basic topological structures of ordinary differential equations. Dordrecht: Kluwer Acad. Publ., 1998.

9. Филиппов В.В. О дифференциальных включениях второго порядка // Фундамент. и прикл. матем. 1997. Т. 3. № 2. С. 587-623.

10. Федорчук В. В., Филиппов В. В. Общая топология. Основные конструкции. М.: Изд-во МГУ, 1988.

11. Филиппов В. В. О теории задачи Коши для обыкновенного дифференциального уравнения с разрывной правой частью // Матем. сб. 1994. Т. 185. № 11. С. 95-118.

12. Филиппов B. В. Что лучше в теории краевых задач для обыкновенных дифференциальных уравнений, метод Лерэ-Шаудера или сдвиг вдоль траекторий? // Дифференц. уравнения. 2001. Т. 37. № 8. С. 1049-1062.

13. Gossez J.-P., Omari P. Nonresonance with respect to the Fučik spectrum for periodic solutions of second order ordinary differential equations // Nonlinear Anal. 1990. V. 14. P. 1079-1104.

14. Gossez J.-P., Omari P. Periodic solutions of a second order ordinary differential equation: a necessary and sufficient condition for nonresonance // J. Differential Equations. 1991. V. 94. № 1. P. $67-82$.

15. Habets P., Omari P., Zanolin F. Nonresonance conditions on the potential with respect to the Fučik spectrum for the boundary value problem // Rocky Mountain J. Math. 1995. V. 25. № 4. P. 1305-1340.

16. Филиппов В.В. О существовании периодических решений // Матем. заметки. 1997. T. 61. № 5. C. 769-784.

Московский государственный

Поступила в редакцию

университет им. М.В. Ломоносова

18.06 .2001 